\title{
The effect of cycling hypoxia on MCF-7 cancer stem cells and the impact of their microenvironment on angiogenesis using human umbilical vein endothelial cells (HUVECs) as a model
}

\author{
Fuad M Alhawarat ${ }^{1}$, Hana M Hammad ${ }^{1}$, Majd S Hijjawi ${ }^{2}$, Ahmad S Sharab ${ }^{2}$, Duaa A Abuarqoub ${ }^{1}$, \\ Mohammad A Al Shhab ${ }^{2}$, Malek A Zihlif ${ }^{\text {Corresp. } 2}$ \\ 1 Department of Biological Sciences, School of Science, The University of Jordan, Amman, Jordan \\ 2 Department of Pharmacology, School of Medicine, The University of Jordan, Amman, Jordan \\ Corresponding Author: Malek A Zihlif \\ Email address: m.zihlif@ju.edu.jo
}

Background: Breast cancer is the most common type of cancer among females. Hypoxia mediates cancer hallmarks and results from reduced oxygen level due to irregularities in tumor vascularization or when the tumor size prevents oxygen diffusion and triggers angiogenesis to compensate for low oxygen. Cancer stem cells (CSCS) are a rare subpopulation, able to self-renew and to give rise to tumor-initiating cells. It is proposed that CSCs' secretions help to recruit endothelial cells via angiogenic factors to establish tumor vascularization. In the tumor microenvironment, the effect of hypoxia on CSCs and the impact of their secretions on triggering angiogenesis and tumor vascularization remain questionable. In this study, the (3D) CSCs derived from MCF-7 were directly exposed to repetitive long-term cycles of hypoxia to assess its effect on CSCS and then to evaluate the role of the hypoxic CSCs' (CSCS ${ }^{\text {HYP })}$ secretions in angiogenesis using (HUVECs) as a model for tumor neovascularization response.

Methods: CSCs derived from MCF-7 cell-line were expanded under repetitive, strictly optimized, longterm/continuous and intermittent hypoxic shots for almost four months to assess hypoxic effect on CSCs, sorted based on CD44 ${ }^{+}$CD24- biomarkers. Hypoxic phenotype of CSCs ${ }^{\text {HYP }}$ was evaluated by assessing the acquired chemoresistance using MTT assay and elevated stemness properties were assessed by flow cytometry. To evaluate the effect of the secretions from CSCs ${ }^{\text {HYP }}$ on angiogenesis, HUVECs were exposed to $\mathrm{CSCs}^{\mathrm{HYP}}$ conditioned-medium (CdM) -in which CSCs had been previously grown - to mimic the tumor microenvironment and to assess the effect of the secretions from CSCs ${ }^{\text {HYP }}$ on the HUVECs' capability of tube formation, migration and wound healing. Additionally, co-culture of CSCs ${ }^{\mathrm{HYP}}$ with HUVECS was performed.

Results: CSCS ${ }^{\mathrm{HYP}}$ acquired higher chemoresistance, increased stemness properties and obtained greater propagation, migration, and wound healing capacities, when compared to CSCs in normoxic condition $\left(\mathrm{CSCs}^{\mathrm{NOR}}\right)$. HUVECs' tube formation and migration abilities were mediated by hypoxic (CSCs) conditioned media (CdM).

Discussion: This study demonstrates that chemoresistant and migrational properties of CSCs are enhanced under hypoxia to a certain extent. The microenvironment of CSCs ${ }^{\mathrm{HYP}}$ contributes to tumor angiogenesis and migration. Hypoxia is a key player in tumor angiogenesis mediated by CSCs. 
1 The effect of cycling hypoxia on MCF-7 cancer stem

2 cells and the impact of their microenvironment on

3

\section{angiogenesis using human umbilical vein endothelial cells (HUVECs) as a model}

\author{
Fuad M Alhawarat ${ }^{1}$, Hana M Hammad ${ }^{1}$, Majd S. Hijjawi², Ahmad S Sharab², Duaa A \\ Abuarqoub $^{1}$, Mohammad A Al Shhab², Malek A Zihlif ${ }^{2}$ \\ Department of Biological Sciences, School of Science, The University of Jordan, Amman 11942, Jordan \\ Department of Pharmacology, School of Medicine, The University of Jordan, Amman 11942, Jordan
}

Corresponding author

Malek Zihlif

m.zihlif@ju.edu.jo

\section{ABSTRACT}

Background: Breast cancer is the most common type of cancer among females. Hypoxia mediates cancer hallmarks and results from reduced oxygen level due to irregularities in tumor vascularization or when the tumor size prevents oxygen diffusion and triggers angiogenesis to compensate for low oxygen. Cancer stem cells (CSCs) are a rare subpopulation, able to selfrenew and to give rise to tumor-initiating cells. It is proposed that CSCs' secretions help to recruit endothelial cells via angiogenic factors to establish tumor vascularization. In the tumor microenvironment, the effect of hypoxia on CSCs and the impact of their secretions on triggering angiogenesis and tumor vascularization remain questionable. In this study, the (3D) CSCs derived from MCF-7 were directly exposed to repetitive long-term cycles of hypoxia to assess its effect on CSCs and then to evaluate the role of the hypoxic $\mathrm{CSCs}$ ' $\left(\mathrm{CSCs}^{\mathrm{HYP}}\right)$ secretions in angiogenesis using (HUVECs) as a model for tumor neovascularization response. 
30 Methods: CSCs derived from MCF-7 cell-line were expanded under repetitive, strictly 31 optimized, long-term/continuous and intermittent hypoxic shots for almost four months to assess 32 hypoxic effect on $\mathrm{CSCs}$, sorted based on $\mathrm{CD} 44^{+} / \mathrm{CD} 24-$ biomarkers. Hypoxic phenotype of $33 \mathrm{CSCs}^{\mathrm{HYP}}$ was evaluated by assessing the acquired chemoresistance using MTT assay and elevated 34 stemness properties were assessed by flow cytometry. To evaluate the effect of the secretions from $35 \mathrm{CSCs}^{\mathrm{HYP}}$ on angiogenesis, HUVECs were exposed to $\mathrm{CSCs}^{\mathrm{HYP}}$ conditioned-medium (CdM) -in which 36 CSCs had been previously grown - to mimic the tumor microenvironment and to assess the effect of the 37 secretions from CSCs ${ }^{\mathrm{HYP}}$ on the HUVECs' capability of tube formation, migration and wound healing.

38 Additionally, co-culture of CSCs ${ }^{\mathrm{HYP}}$ with HUVECs was performed.

Results: $\mathrm{CSCs}^{\mathrm{HYP}}$ acquired higher chemoresistance, increased stemness properties and obtained greater propagation, migration, and wound healing capacities, when compared to CSCs in normoxic condition $\left(\mathrm{CSCs}^{\mathrm{NOR}}\right)$. HUVECs' tube formation and migration abilities were mediated by hypoxic (CSCs) conditioned media (CdM).

Discussion: This study demonstrates that chemoresistant and migrational properties of CSCs are enhanced under hypoxia to a certain extent. The microenvironment of $\mathrm{CSCs}^{\mathrm{HYP}}$ contributes to tumor angiogenesis and migration. Hypoxia is a key player in tumor angiogenesis mediated by CSCs.

\section{INTRODUCTION}

Cancer Stem Cells (CSCs) are subpopulation in the cancer tissue that slowly divide with an ability to regenerate by uneven cell division (Huang \& Rofstad 2017), contributing to cancer relapse, invasiveness and thus higher cancer mortality (Peitzsch et al. 2017). CSCs have the same intrinsic features as normal stem cell populations found throughout the body, including the ability of self-renewal and differentiation (Batlle \& Clevers 2017). In most solid tumors, including breast carcinoma, hypoxia is a mutual hallmark that is proven to associate with poor prognosis of cancer patients (Muz et al. 2015). Various stress-response pathways that drive cancer cells toward a self-stabilizing and anti-apoptotic phenotype are activated upon the exposure of cells to sub-physiologic concentrations of oxygen $\left(\leq 1 \% \mathrm{O}_{2}\right)$ or hypoxia (Crowder et al. 2014). Hypoxia stabilizes the hypoxia-inducible factor- $\alpha$ (HIF $\alpha$ ) proteins that contribute to various pro-survival processes in cancer cells, such as angiogenesis, abnormal proliferation and metabolic alterations (Bristow \& Hill 2008; Kietzmann et al. 2016; Kong et al. 2014), and thus increases the expression of pro-survival factors that support radioresistance and chemoresistance (Crowder et al. 2014; Senthebane et al. 2017). The exact signaling pathways by which hypoxia activates its effects are complex and remain under investigation. Among wellrecognized mechanisms are elevated genomic instability and irregular cell division (Crowder et 
64 al. 2014; Hammer et al. 2007), uncontrolled reactive oxygen species and redox mechanisms, 65 aerobic glycolysis metabolic shift (Denko 2008), and reduced expression caused by 66 proapoptotic-factors (Gordan et al. 2008).

67 Cycling hypoxia determines the state of oxygen level and its dispersal in solid tumor tissues.

68 Dysfunctional heterogenic blood supply and tumor irregular vascularity in cancer cause oxygen

69 fluctuation for irregular periods with intermittent re-oxygenation intervals (Brurberg et al. 2004;

70 Muz et al. 2015). Acute hypoxia is an immediate brief exposure to a short-term hypoxic status

71 where the blood vessel occlusion lasts for at least few minutes and up to 72 hours (Chaplin et al.

72 1987; Muz et al. 2015). These changes in the bloodstream and the limited oxygen availability

73 result in chronic hypoxia, which is more apparent in large-sized tumors and energizes long-term

74 cellular changes (Eales et al. 2016). In experimental protocols, chronic hypoxic exposure is

75 obtained by incubating cells in hypoxia up to several weeks (Cassavaugh \& Lounsbury 2011).

76 Indeed, clinical responses to cancer therapy are directly affected by both chronic and acute

77 hypoxic sections in solid tumors, inducing their growth, metastatic ability, and cell death 78 resistance (Al-Hajj et al. 2003).

79 Angiogenesis, which is defined as tumor neovascularization is essential for tumor development 80 and maintenance (Kaur et al. 2005; Zhao \& Adjei 2015). It is also considered an important 81 cellular parameter of tumorigenesis (Wang et al. 2015). Elevated levels of vascular endothelial 82 growth factors (VEGFs) are expressed by endothelial cells involved in angiogenesis and bone 83 marrow-derived progenitor cells, and moreover, many types of human cancer cells can also 84 secrete VEGFs (Kerbel 2008). Evidence from different studies indicates that CSCs show greater 85 potential for tumor initiation, generation, and production of higher levels of VEGFs than the non86 stem cells in cancer population, thus exhibiting more potent proangiogenic capability (Ping \& 87 Bian 2011; Wang et al. 2016). CSCs with elevated VEGF levels are typical example for the 88 significance of angiogenesis in tumor progression (Ojha et al. 2017).

89 In conclusion, CSCs' secretions help to recruit endothelial cells via angiogenic factors to 90 establish tumor vascularization. Moreover, hypoxia triggers angiogenesis in cancer tissues. The 91 studies that investigate the effect of long-term hypoxia on CSCs are extremely rare, so the cross92 talk between long-term hypoxia and its effect on CSCs and their role in angiogenesis remains 93 questionable. In this context, we hypothesized that repetitive exposure to cycles of 94 hypoxia/normoxia conditions may be a driving force that promotes a highly resistant and 95 invasive CSCs subpopulation, and CSCs are proposed to generate higher levels of tumor 96 vascularization, due to induced angiogenesis, than regular cancer tissues. 
97 Accordingly, the present study aims at investigating the effect of long-term hypoxia on CSCs 98 and the impact of hypoxic $\mathrm{CSCs}\left(\mathrm{CSCs}^{\mathrm{HYP}}\right)$ secretions on angiogenesis in the tumor 99 microenvironment. CSCs were derived from MCF7 cell-line and were expanded under repetitive 100 strictly optimized long-term and intermittent hypoxic shots to assess the effect of hypoxia on 101 them. Both intermittent and long-term hypoxia were directly applied for almost four months on 102 sorted and identified CSCs as (3D) mammospheres. Thereafter, to assess the impact of secretions

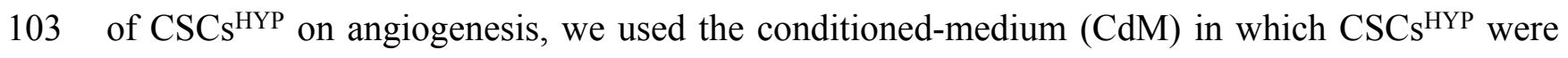
104 expanded and grown -as it comprises the $\mathrm{CSC}^{\mathrm{HYP}}$ secretions- to treat HUVECs and then examine 105 their angiogenic ability.

\section{MATERIALS AND METHODS}

Workflow for the methodology adopted in this study is summarized in (Figure 1).

\section{Culture Conditions}

111 The breast cancer cell line (MCF-7) was purchased from the American Type Culture Collection

112 (ATCC, USA), and was expanded as a monolayer in vented $75 \mathrm{~cm}^{2}$ cell culture flasks (Membrane

113 Solutions, USA) using RPMI 1640 media (HyClone, USA). The media was supplemented with 114 heat-inactivated fetal bovine serum (FBS) 10\% (v/v) (HyClone, USA), antibiotics (100 $\mu \mathrm{g} / \mathrm{ml}$ 115 streptomycin and 100U/ml penicillin) (HyClone, USA), and 1\% 2mM L-glutamine (HyClone, 116 USA). The cells were incubated in a designated incubator (Nuaire, China) at $\left(5 \% \mathrm{CO}_{2}\right.$ at $\left.37^{\circ} \mathrm{C}\right)$.

117 All cell culture procedures were performed in sterile conditions under a class II biological safety

118 cabinet (Heal-Force, China). All used materials and disposables were disinfected with ethanol $11976 \%$ before use.

120

\section{Mammosphere Cultures}

122

123 To obtain CSCs from MCF-7 cell line and propagate them as mammospheres, MCF-7 cells were 124 expanded in complete growth media described above as a single-cell suspension in a standing 125 flask to enhance the growth of CSCs as they tend to grow in non-adherent conditions. Two days 126 later, remaining cells were collected by centrifugation, washed in phosphate buffer saline, and 127 seeded in 6 well ultra-low adherence plates (Corning, NY) at a density of 10,000 cells/well. Cells 128 were expanded in serum-free DMEM/F12 (Sigma-Aldrich) media, supplemented with $20 \mathrm{ng} / \mathrm{mL}$ 129 basic fibroblast growth factor (GIBCO, USA), $20 \mathrm{ng} / \mathrm{mL}$ epidermal growth factor (MPBio, 130 USA), 2\% B27 (GIBCO, USA), $10 \mu \mathrm{g} / \mathrm{mL}$ insulin (MPBio, USA), 0.5 $\mu \mathrm{g} / \mathrm{mL}$ hydrocortisone 
131 (Nacalai, Japan), 0.4\% bovine serum albumin (Sigma, USA), and $2 \mathrm{mM}$ L-Glutamine (Biowest

132 Co., France). This is called mammospheres culture media. Cells were preserved in a humidified

133 incubator at $37{ }^{\circ} \mathrm{C}, 5 \% \mathrm{CO}_{2}$. Every two days, fresh media $(2 \mathrm{~mL})$ were added to each well

134 (without removal of the old one). At day seven, primary mammosphere clusters were counted,

135 then collected and centrifuged to form a pellet. In order to obtain single cells, the pellet was

136 enzymatically disaggregated with trypsin and dispersed by passing through a $40 \mu \mathrm{m}$ pore-size

137 filter (Millipore, Billerica, MA). Single cells of primary mammospheres were collected for

138 sorting, using beads technique and identified by flow cytometry (detailed below) before and after

139 sorting. Disaggregated cells were cultured in ultra-low adherence flask (Corning, NY) instead of

140 well plates to obtain secondary mammospheres. Then, the same procedure was applied again to

141 obtain tertiary and quaternary mammospheres.

142 To calculate the percentage of mammosphere forming efficiency (\%MFE), the number of

143 mammospheres was divided by the number of seeded cells and multiplied by hundred.

144 Mammospheres were counted under 10x magnification of an inverted light microscope

145 (Olympus, USA). Twenty one (21) days after initial seeding in ultra-low attachment and serum-

146 free media, a sufficient mammospheres' population was established.

147

148 Cell Viability

149 Cellular viability was evaluated using the trypan blue method. After de-attachment and good

150 homogenization of cells until there was a single-cell suspension, a sample was taken and diluted

151 in 1:1 ratio with trypan blue (Sigma-Aldrich, UK). Then 10uL of the diluted mixture was loaded

152 on a hemocytometer (Neubauer Double, Zuzi, Spain) and inspected under an inverted light

153 microscope (Olympus, USA). Cells with shiny white appearance were considered viable and

154 counted because their cell membrane was impermeable to trypan blue, while blue-colored cells

155 were disregarded. Hypoxic cells' viability was checked upon every other split and compared to

156 its normoxic counterparts to assess proliferation changes. While mammosphere cells were

157 enzymatically disaggregated into single-cells in order to observe viability (Cassavaugh \&

158 Lounsbury 2011).

159

160 CSCs Sorting with Magnetic Beads

$161 \mathrm{CD}^{+} / \mathrm{CD} 24^{-}$are the expression biomarkers of interest in MCF-7 derived mammospheres. At

162 day seven of the initial culture of mammospheres in 6-well plate, CSCs enriched with

$163 \mathrm{CD} 44^{+} / \mathrm{CD} 24^{-}$were sorted from MCF-7 mammospheres using MagCellect CD24-/CD44 ${ }^{+}$Breast

164 Cancer Stem Cell Isolation Kit (R\&D System, Cat. MAGH111) according to their protocol. 
165 Initially, CD24+ cells were labeled and removed magnetically. Then from the CD24- population,

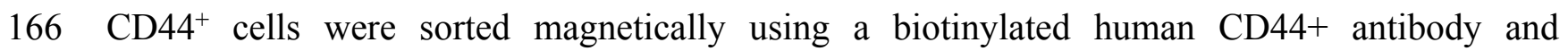

167 streptavidin-conjugated magnetic beads as a positive selection model. The efficiency of sorting

168 was assessed by staining recovered cells with fluorochrome-conjugated anti-human $\mathrm{CD}_{4} 4^{+}$and

$169 \mathrm{CD}^{-} 4^{-}$antibodies. Isolated CD $44^{+} / \mathrm{CD} 24^{-}$cells were later identified via FACS analysis.

170

\section{Exposure to Hypoxia}

172 To create hypoxic conditions, an anaerobic atmosphere generating system, AnaeroGen Compact

173 (Oxoid, UK) was used. The AnaeroGen system is used in microbiology area to create the low

174 oxygen conditions needed for the growth of anaerobic bacteria. Here, we adopted the AnaeroGen

175 system to induce hypoxia into CSCs. The system consists of a gas generatingsachets and tightly

176 sealable bags. The sachet reacts promptly upon contact with air and consumes oxygen thus

177 reducing oxygen concentration to less than $1 \%$ inside the bag. It has been confirmed in previous

178 studies by using biochemical and electronic testing that this technique reduces oxygen content in

179 plastic bag to below 1\% within 30 minutes (Chenevier-Gobeaux et al. 2013; Mellor et al. 2005).

180 The vented ultra-low attachment $75 \mathrm{~cm}^{2}$ tissue culture flasks were placed inside the plastic bags

181 and the sachets were placed inside then sealed. After 21 days of culturing the mammosphere

$182 \mathrm{CSCs}$, they were divided into two groups and exposed to hypoxic conditions intermittently

183 (INTR.) or continuously (CONT.). CSCs in the intermittent group were exposed to 10, 20, 30 or

18440 hypoxic shots for 8 hours 3 times a week, denoted as (INTR.10, INTR.20, INTR.30, and

185 INTR.40) respectively, while CSCs in the continuous group was exposed to 5, 10, or 15 hypoxic

186 shots for 72 hours once per week, denoted as (CONT.5, CONT.10, and CONT.15). Apart from

187 these optimized hypoxic shots, CSCs mammospheres were also incubated in normoxic

188 conditions as control ( $\mathrm{CSCs}^{\mathrm{NOR}}$ ), using the same culture media and conditions that were used for

189 expansion of mammospheres detailed above.

190

191 Flow Cytometry to Examine Enrichment of CSCs Mammosphere with CD44 ${ }^{+}$CD24- under

\section{Hypoxia/Normoxia}

193 Flow cytometry was used to identify and characterize CD44 ${ }^{+}$CD24- surface phenotype, which is

194 in direct proportion to stemness, and was performed for parental MCF-7 cells, un-sorted

195 mammospheres, sorted CSCs mammospheres three days after sorting, CSCs mammospheres

196 which were 21 days-old all under normoxic conditions and finally for CSCs ${ }^{\mathrm{HYP}}$ exposed to shots

197 of INTR.20, INTR.40, CONT.5 and CONT.15. Mammospheres were dispersed to obtain

198 single-cell populations as described above. The CSCs' mammosphere pellet was washed in 
199 phosphate-buffer saline (PBS) (HyClone, USA) with 2\% bovine serum albumin and stained with 200 APC-anti-mouse CD24 and PE-anti-mouse CD44 antibodies (BD Pharmingen, CA). CSCs 201 mammospheres were incubated in ice for 30 minutes, washed twice with PBS and then fixed in 202 PBS containing paraformaldehyde. The flow cytometric analysis was performed on a BD 203 FACSCalibur system (BD Biosciences, CA), and the identification was performed with the BD 204 Cellquest software (BD Biosciences).

206 Cytotoxicity Evaluation Using MTT Assay

207 To examine the chemoresistance of MCF-7 parental cells and their derived CSCs, both CSCs ${ }^{\mathrm{NOR}}$

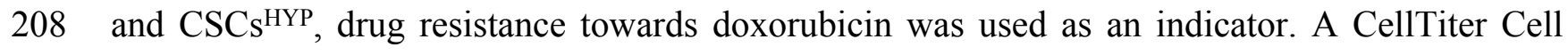
209 Proliferation Assay Kit ${ }^{\circledR}$ (Promega, Madison, USA) was used. This assay includes 3-(4,5210 Dimethylthiazol-2-yl)-2,5-diphenyl tetrazolium bromide (MTT) which is a yellow tetrazole 211 reduced by viable cells to purple formazan. Consequently, surviving cells 
213 after being exposed to multiple concentrations of doxorubicin can be quantified by 214 spectrophotometry. The MTT proliferation assay was performed on MCF-7 parental cells seeded 215 as a monolayer in an adherent 96-well plate. Moreover, $\mathrm{CSCs}^{\mathrm{HYP}}$ were dissociated into single216 cells, filtered, counted then seeded at a $7 \times 10^{3}$ cells/well density in a 96-well plate and incubated

217 for 24 hours before treatment. Each concentration was tested in triplicate on CSCs exposed to 218 (INTR.10, INTR.20, INTR.30, and INTR.40) or exposed to (CONT.5, CONT.10, and 219 CONT.15). A control experiment was done for sorted and identified CSCs ${ }^{\mathrm{NOR}}$ from 220 mammospheres cultured under normoxic conditions.

221 Incremental serial dilutions of doxorubicin concentrations from $0.01 \mu \mathrm{M}$ to $200 \mu \mathrm{M}$ were used. 222 After 72 hours of incubation, old media was aspirated, and $100 \mu \mathrm{l}$ of fresh media was added to 223 each well. Then, $15 \mu \mathrm{l}$ of MTT reagent was added to each well and incubated for 4 hours. 224 Solubilization/stop solution was added to each well and incubated for an hour. Absorbancy was 225 read using an Elisa reader (Sunrise basic sciences, Austria) at $570 \mathrm{~nm}$ wavelength. The half 226 maximal inhibitory concentration $\left(\mathrm{IC}_{50}\right)$ was calculated using GraphPad PRISM ${ }^{\circledR} 7.04$ software 227 (GraphPad Software, Inc.).

\section{Endothelial Cells Isolation from Umbilical Cords}

230 Human umbilical cords were obtained from two delivering females at the Jordan University 231 Hospital after getting the approval of their Institutional Review Board (IRB), decision number 232 98/2018 and approval number (67/2018/481) and both patients having signed an informed 233 consent before sample collection.

234 Enzyme digestion technique was used to isolate endothelial cells from the human umbilical cord. 235 Debris on the outer surface of the cord was wiped using antiseptic solution. Any clots formed 236 inside the vein were removed by slight squeezing. The umbilical vein was washed with RPMI 237 medium (HyClone, USA) containing 1\% penicillin/streptomycin (HyClone, USA) to remove 238 excess blood and debris using $20 \mathrm{~mL}$ syringe inserted into the cord. Then, $0.1 \%$ of type I 239 collagenase (Sigma-Aldrich, USA) was put inside the vein where it was incubated for 20 minutes 240 at $37^{\circ} \mathrm{C}$ for digestion. Hereafter, the vein was washed with $20 \mathrm{~mL}$ RPMI media (HyClone, USA) 241 to stop collagenase proteolytic activity and was centrifuged at $1500 \mathrm{rpm}$ for $5 \mathrm{~min}$. The resultant 242 pellet was resuspended in $5 \mathrm{ml}$ endothelial cell growth medium EGM-2 media (Lonza, 243 Walkersville, MD, USA) which consists of the endothelial cell basal media EBM-2 with 10\% 244 FBS and other additives (including VEGF, bFGF- Fibroblast Growth Factor, EGF-Epidermal 245 Growth Factor, and IGF-Insulin Growth Factor). HUVECs were expanded on 0.2\% gelatin246 coated T25 tissue culture flask and incubated overnight. Next day media was renewed to get rid 247 of cells debris. Only passages earlier than 6 of HUVECs were used in all experiments. 
249 Collection of Conditioned-Medium From Hypoxic/Normoxic CSCs

250 In order to obtain the conditioned-medium (CdM), CSCs were seeded as single cells at a density 251 of 10,000 cells $/ \mathrm{cm}^{2}$ in an ultra-low adherence $75 \mathrm{~cm}^{2}$ flasks with serum-free DMEM/F-12 and 252 with supplements (mammospheres culture media detailed above). CSCs mammospheres were 253 starved overnight by washing them twice with serum and supplement free DMEM/F-12 media, 254 then centrifuged at $300 \mathrm{rpm}$ for 10 minutes. Finally, the media was renewed without serum and 255 without supplements for 8 hours for intermittent and 72 hours for the continuous hypoxic 256 condition. The resultant CdM from where CSCs grown under hypoxia or normoxia were 257 collected, centrifuged at $300 \mathrm{rpm}$ for $10 \mathrm{~min}$ to remove cellular components and filtered through $2580.2 \mu \mathrm{m}$ pore-size filters (Millipore, Billerica, MA). CdM from flasks under normoxic conditions 259 were used as a control. Aliquots of the $\mathrm{CdM}$ were stored at $-80^{\circ} \mathrm{C}$ before being used. $\mathrm{CdM}$ were 260 collected from eight sources, from $\mathrm{CSCs}^{\mathrm{NOR}}$, and from $\mathrm{CSCs}^{\mathrm{HYP}}$ grown under the hypoxic shots 261 (INTR.10, INTR.20, INTR.30, INTR.40, CONT.5, CONT.10, or CONT.15) and were utilized 262 in successive experiments as discussed subsequently.

263

264 Capillary-Like Tube Structure Formation Assay using CSCs' Mammospheres (CdM) on 265 HUVECS

266 The effect of the factors secreted in the CdM obtained from $\mathrm{CSCs}^{\mathrm{HYP}}$ and from $\mathrm{CSCs}^{\mathrm{NOR}}$ on the 267 angiogenic ability of HUVECs was examined through the formation of capillary tube-like 268 structures by HUVECs, using the in vitro matrigel angiogenesis assay to mimic the 269 microenvironment of CSCs. To eliminate the impact of growth factors in Matrigel, reduced 270 growth factor Matrigel (BD Biosciences, USA) was used. HUVECs were incubated for 6 hours 271 in endothelial basal medium (EBM-2, Lonza) without serum and supplements for starvation. 272 Briefly, the Matrigel stored at $-20^{\circ} \mathrm{C}$ was thawed in ice to prevent premature polymerization. 273 Aliquots of $50 \mu \mathrm{l}$ were plated into each well of pre-chilled 96-well culture plates and were left to 274 polymerize at $37^{\circ} \mathrm{C}$ for 2 hours. HUVECs were removed from confluent cultures by treatment 275 with Trypsin $0.05 \%$ (HyClone, USA). HUVECs were collected using serum-containing media to 276 stop trypsin effect, then counted, centrifuged and washed with phosphate buffer saline, then 277 resuspended with CdM obtained from the different hypoxic episodes, including intermittent shots 278 (INTR.10, INTR.20, INTR.30, INTR.40) and continuous shots (CONT.5, CONT.10, 279 CONT.15) of hypoxia and from its normoxic counterparts as a control in triplicates. HUVECs 280 were seeded at a density of $2 \times 10^{4}$ cells/well. For quantification of tube formation complex, three 281 primary variables were used to determine the magnitude of tube formation, average of total 
282 length of the branched tube, the number of loops and covered area. These variables were 283 measured by Wimasis Image Analysis software. 
286 The CdM that was collected as described above, was used for the determination of VEGF levels 287 secreted by $\mathrm{CSC}^{\mathrm{HYP}}$ and $\mathrm{CSC}^{\mathrm{NOR}}$ using the Enzyme-Linked Immunosorbent kit. Human VEGF 288 quantitative ELISA assay (R\&D Systems, Minneapolis, MN) was performed according to 289 manufacturer's instructions. A quantity of 200ul of the CdM from different $\mathrm{CSCs}^{\mathrm{HYP}}$ treatments was tested.

291

\section{Cell Migration Assay of HUVECs}

293

294

HUVECs ability to migrate under the treatment of the CdM was investigated using a Transwell plate of 8 um pore-size chamber (Corning, USA). HUVECs were starved overnight using EBM2 media serum and supplement free for 6 hours, then seeded in the upper chamber (insert) of the 24 well plate at density of $5 \times 10^{4}$ cells in $500 \mathrm{uL}$ of EBM -2 media, each placed into lower wells containing $750 \mathrm{ul}$ of CdM obtained from (INTR.10, INTR.20, INTR.30, INTR.40) intermittent hypoxic shots in addition to continuous hypoxic shots (CONT.5, CONT.10, CONT.15). The same procedure was applied to the control by using serum-free EBM-2 media in the lower well instead of CdM. Then, cells were allowed to migrate for 8 hours, then $70 \%$ cold ethanol was used for 10 minutes to fix the chambers. Membranes were stained with $0.5 \%(\mathrm{w} / \mathrm{v})$ crystal violet (Sigma-Aldrich, St. Louis, MO, USA) for 30 minutes and afterward washed carefully with water. Cotton swabs were used to remove the cells that were not able to migrate to lower wells. Solubilizing bound crystal violet in methanol $100 \%$ (wt./vol) was used to quantify the migrated cells. Cells were inspected under an inverted light microscope (Olympus, USA) and photographed at $4 \mathrm{x}$ magnification. Each sample was done in triplicate and three fields were measured from each to calculate the migration.

\section{Direct and Indirect Co-culture of HUVECs and CONT.5 CSCs Mammospheres}

311

312 A co-culture system has been used to better understand how $\mathrm{CSCs}^{\mathrm{HYP}}$ regulate their

313 microenvironment and interact with HUVECs as a vascularization model for angiogenesis and 314 migration.

315 Direct Co-culture:

316 HUVECs were seeded on $0.1 \%$ gelatin (Sigma, USA) coated 6-well plate until $40 \%$ confluency 317 in EGM-2 media. From the $\mathrm{CSCs}^{\mathrm{HYP}}$ subpopulation exposed to CONT.5, a single hypoxic CSC 
318 was co-cultured in contact with HUVECs. The plates were incubated for few hours to allow

$319 \mathrm{CSCs}^{\mathrm{HYP}}$ adhere to the wells already seeded with HUVECs. Media was changed to a 1:1 mixture

320 of EBM-2 and DMEM/F-12 free from serum and supplements and incubated for 72 hours.

321 Indirect Co-culture:

322 Transwell plates of $0.4 \mu \mathrm{m}$ pore size chambers were seeded with HUVECs in lower wells after

323 coating them with $0.1 \%$ gelatin, while $\mathrm{CSCs}^{\mathrm{HYP}}$ that were exposed to CONT.5 were seeded in

324 the upper chamber as co-cultured in a 1:1 mixture of EBM-2 and DMEM/F-12 free from serum

325 and supplements.

326 Control HUVECs were cultured in EBM-2 without serum and without supplements in both the

327 direct and indirect experiments.

328

329

Wound Healing Assay for HUVECs

330

In a 6 -well plate, HUVECs at a density of $12 \times 10^{4}$ were seeded till confluency. Then they were

331 incubated for 6 hours in EBM-2 media without serum to prevent any external factors from contributing to wound closure. After 6 hours, using a 200uL micropipette tip, a straight woundlike scratch was made into the cells. Then cells were treated with $\mathrm{CSCs}^{\mathrm{HYP}} \mathrm{CdM}$ obtained from INTR.10, INTR.20, INTR.30, INTR.40, CONT.5, CONT.10 and CONT.15 hypoxic shots in which $\mathrm{CSCs}^{\mathrm{HYP}}$ were grown, in addition to the control which was treated with $\mathrm{CdM}$ obtained from $\mathrm{CSCs}^{\mathrm{NOR}}$. Cells were carefully washed using phosphate buffer saline (HyClone, USA), before and after making the scratch. Wound healing was inspected under an inverted light microscope (Olympus, USA) and photographed after 48 hours and at the beginning of the experiment. The surface area of the wound could not be calculated by image analysis software because control cells started to die after 16 hours.

341

\section{Wound Healing Assay for Hypoxic CSCs}

In a 6-well plate coated with $1 \%$ gelatin, CSCs were seeded at a density of $8 \times 10^{4}$ and incubated overnight, using DMEM/F-12 media without serum to prevent any external factors from contributing to wound closure. The subpopulation of CSCs used was originally cultured under intermittent or continuous hypoxic conditions; INTR.10, INTR.20, INTR.30, INTR.40, normoxia. They were all used upon confluency before being seeded in a gelatin-coated 6-well plate to allow CSCs adherence as a 2D monolayer for wound healing assay. Using a 200uL 
35212 without serum. Wound healing was photographed at the beginning of the experiment and after

35336 hours. The surface area of the wound was measured by image analysis (ImageJ 1.4.3.67

354 Launcher Symmetry Software).

355 
356 Statistical Analysis

357 Results were presented as a mean \pm SD. Experiments were performed in triplicate with 358 comparable results unless indicated otherwise. An ANOVA test was used to compare the 359 differences between replicates which were considered significant at $\mathrm{p} \leq 0.05$. GraphPad Prism 360 (version 7.04, San Diego, CA) was used for statistical analysis.

\section{RESULTS}

364

(3D) Mammospheres Derived from MCF-7 cells

366

367

368

369

370

371

372

373

374

375

376

377

378

379

380

381

382

383

384

385

386

387

388

Mammosphere culture has been widely used to enrich CSCs. Thereof, MCF-7 mammospheres were cultured in ultra-low adherence plates and flasks, in serum-free media with supplements and successfully formed rigid and compact (3D) structures within 3-7 days (Figure 2). The mammosphere multicellular spheroidal clumps became larger in size at day 7 compared to earlier days of growth as shown in (Figure 2) with 30 um scale bar comparison. Moreover, as a quantitative measure, at day seven the mammosphere formation efficiency (MFE) of MCF-7 cells was $0.012 \%( \pm 0.002 \%)$ only and after three weeks the MFE had increased to 5.30\% ( \pm $0.307 \%)$.

\section{Sorting of CSCs From 3D Mammospheres Using Magnetic Beads}

The magnetic beads separation protocol uses antibodies conjugated to a magnetic bead. CSCs were successfully sorted and isolated using the magnetic beads technique, but their number became sufficient only 3 days after sorting. Thereafter, sorted CSCs were identified via FACS analysis and were used for hypoxic-condition exposure. The expression of $\mathrm{CD}^{+} 4^{+} / \mathrm{CD} 24^{-}$ mammospheres is shown in (Table 1) and their morphology is shown in (Figure 2).

\section{Enrichment of sorted CSCs mammospheres under Hypoxic/Normoxic Conditions}

The morphology of $\mathrm{CSCs}^{\mathrm{HYP}}$ and the control in normoxia CSCs ${ }^{\mathrm{NOR}}$ is shown in (Figure 3). It is observed that $\mathrm{CSCs}^{\mathrm{HYP}}$ that underwent CONT.5 and INTR.20 hypoxic shots were denser and larger in number than $\mathrm{CSCs}^{\mathrm{NOR}}$. $\mathrm{CSCs}^{\mathrm{HYP}}$ that underwent CONT.15 and INTR.40, that is the maximum and final hypoxic shots showed the least growth compared to other hypoxic exposures and $\mathrm{CSCs}^{\mathrm{NOR}}$. The MFE could not be measured for $\mathrm{CSCs}^{\mathrm{HYP}}$ because long-term hypoxic exposure (INTR.40 and CONT.15) made mammospheres aggregate into clumps, thus, the single 
389 mammosphere could not be distinguished and compared to control and to other hypoxic 390 mammospheres.

391 
392 Identification of CD44 ${ }^{+}$CD24- Phenotype Content by Flow Cytometry

393 Flow cytometry conducted for CSCs after 3 days of sorting showed a percentage of $394 \mathrm{CD}_{4}{ }^{+} / \mathrm{CD} 24^{-}$expression equal to 81.0 ( $\pm 7.5 \%$ ). Three weeks (21 days) after growing in ultra395 low attachment flasks, the percentage decreased to approximately $35.5( \pm 4.5 \%)$. In contrast, the 396 parental MCF-7 cells had only $1( \pm 0.1 \%)$ expression and the unsorted mammospheres had 33.2 $397( \pm 3 \%)$ expression. After exposure to hypoxic conditions, flow cytometry results based on $398 \mathrm{CD}^{+} 4^{+} \mathrm{CD} 24^{-}$markers showed that the highest expression occurred in $\mathrm{CSCs}^{\mathrm{HYP}}$ exposed to 399 INTR.20 shots, $39.8( \pm 5.2 \%)$, and in $\mathrm{CSCs}^{\mathrm{HYP}}$ exposed to CONT.5, $51.6( \pm 6.1 \%)$. On the other 400 hand, the lowest percentages were observed in $\mathrm{CSCs}^{\mathrm{HYP}}$ that underwent or were exposed to 401 INTR.40 shots, $0.3( \pm 0.01 \%)$, and in $\mathrm{CSCs}^{\mathrm{HYP}}$ exposed to CONT.15, $0.5( \pm 0.05 \%)$. In 402 conclusion, isolated and sorted $\mathrm{CD} 44^{+} / \mathrm{CD} 24^{-}$breast cancer cells only temporarily preserve this 403 phenotype and ultimately revert to an equilibrium state in which the expanded sub-population 404 regains the original cell surface profile of the parental cell line, which was deduced because the 405 unsorted mammospheres and the 3-week old sorted CSCs both have similar $\mathrm{CD}_{4} 4^{+} / \mathrm{CD}_{2} 4^{-}$ 406 content $\left(33.2 \%\right.$ and $35.5 \%$, respectively). The $\mathrm{CD} 44^{+} / \mathrm{CD} 24^{-}$percentage in unsorted 407 mammospheres that were grown in ultra-low attachment plates in serum-free media for 7 days 408 before sorting was $33.2( \pm 3 \%)$, which is much higher than the percentage of parental MCF-7 409 cells $1.0( \pm 0.1 \%)$. The expression of $\mathrm{CD} 44^{+} / \mathrm{CD} 24^{-}$content for all subpopulations is summarized 410 in (Table 1) and demonstrated in (Figure 4).

411

412 Hypoxic CSCs Show Higher Drug Resistance to Conventional Chemotherapies

413 To examine whether self-renewing CSCs mammospheres have higher chemoresistance ability, 414 and to assess if hypoxic treatment of CSCs would increase their stemness character and 415 chemoresistance, MTT assay was implemented to test the sensitivity of parental MCF-7 versus 416 the sorted CSCs mammosphere towards doxorubicin. In addition, we assessed the resistance 417 towards doxorubicin acquired by $\mathrm{CSCs}^{\mathrm{HYP}}$ which underwent intermittent hypoxic shots 418 (INTR.10, INTR.20, INTR.30 INTR.40), and $\mathrm{CSCs}^{\mathrm{HYP}}$ that underwent continuous hypoxic 419 shots (CONT.5, CONT.10, CONT.15). Then we compared the inhibitory drug concentration $420\left(\mathrm{IC}_{50}\right)$ results for the $\mathrm{CSCs}^{\mathrm{HYP}}$ versus $\mathrm{CSCs}^{\mathrm{NOR}}$ mammospheres (as control) and compared the $421 \mathrm{IC}_{50}$ value of $\mathrm{CSCs}^{\mathrm{HYP}}$ versus parental MCF-7 cells, described in Table 2. Overall, the drug 422 resistance of $\mathrm{CSCs}^{\mathrm{NOR}}$ was found to be higher $\left(\mathrm{IC}_{50}=1.90 \mathrm{uM}\right)$ when compared to the parental 423 MCF-7 cells $\left(\mathrm{IC}_{50}=0.46 \mathrm{uM}\right)$ by 4.08 times. Comparing the $\mathrm{IC}_{50}$ values of $\mathrm{CSCs}^{\mathrm{NOR}}$ 424 mammospheres with $\mathrm{CSCs}^{\mathrm{HYP}}$, we observed significantly increased values under hypoxic 425 conditions at INTR.20 $\left(\mathrm{IC}_{50}=6.13 \mathrm{uM}\right)$ and CONT.5 $\left(\mathrm{IC}_{50}=7.12 \mathrm{uM}\right)$ with (3.28) and (3.76) fold 
426 increases respectively compared to $\mathrm{IC}_{50}$ of $\mathrm{CSCs}^{\mathrm{NOR}}$ mammospheres. These increaseswere much

427 higher upon comparing $\mathrm{IC}_{50}$ for $\mathrm{CSCs}^{\mathrm{HYP}}$ with parental MCF-7. The lowest $\mathrm{IC}_{50}$ values were 428 observed at INTR.40 and CONT.15 which represent the final hypoxic treatments. Taken 429 together, these results support the likelihood that enriching the mammospheres' culture with 430 CSCs contributed to the higher drug resistance toward doxorubicin when compared to the 431 parental MCF-7.

432

Hypoxic Conditioned Medium (CdM) Induces HUVECs to Form Capillary-Like Tube

\section{Structures in Matrigel}

In this study, HUVECs were treated with CdM in which CSCs were expanded under several hypoxic and normoxic conditions, as described in the method section. Our results showed that treating HUVECs with $\mathrm{CdM}$ obtained from $\mathrm{CSCs}^{\mathrm{HYP}}$ resulted in an increased construction of capillary-like tube structures that reached its peak at 8 hours of treatment and disappeared eventually in a time-dependent manner. However, HUVECs treated with CdM obtained from $\mathrm{CSCs}^{\mathrm{NOR}}$ (control), resulted in a reduced formation of complex tubular structures compared to the ones treated with the $\mathrm{CdM}$ obtained from $\mathrm{CSCs}^{\mathrm{HYP}}$ (Figure 5). The capillary-like tube structures were evaluated by quantitative analysis using Wimasis Image Analysis. The results showed that the highest increase of branched tube lengths, the number of loops and the covered area was with HUVECs that were cultured with $\mathrm{CdM}$ obtained from $\mathrm{CSCs}^{\mathrm{HYP}}$ culture that underwent INTR.20 and CONT.5 shots at each time point (as demonstrated in Figures 5 and 6). A bar graph (data combined from three independent trials) (Figure 6) shows that "the total length of the tubes" underwent a remarkable increase upon the treatment with CdM from INTR.20 and CONT.5, by almost 2.4 fold in both INTR.20 and CONT.5 when compared to the control, moreover, the "covered area" significantly increased when HUVECs were cultured with CdM from $\mathrm{CSCs}^{\mathrm{HYP}}$ culture that underwent CONT.5 and INTR.20, by 3.5 and 2.7 times respectively. The total number of loops were significantly increased by 6.7 fold in INTR.20 and 8.9 fold in CONT.5 compared to control HUVECs incubated with CdM obtained from the CSCs ${ }^{\mathrm{NOR}}$ culture expanded in normoxic state. As shown in (Figures 5 \& 6), CdM induced a significant increase in tube formation in HUVECs starting from the treatment with INTR.10 CdM and reaching a maximum at INTR.20 (Figure 5F), whereas CONT.5 (Figure 5B) caused the highest increase in tube formation.

457

\section{Detection of Secreted VEGF in Conditioned-Medium (CdM) by ELISA}

In order to identify the possible mediators of angiogenesis in breast cancer, a quantitative assay 
460 was performed for the main angiogenic factor, VEGF-A. As the CdM obtained from the hypoxic 461 episodes INTR.20, INTR.30 and CONT.5 had the highest VEGF concentrations, INTR.20, $4621159( \pm 90 \mathrm{pg} / \mathrm{ml})$, INTR.30, $1013( \pm 32 \mathrm{pg} / \mathrm{ml})$ and CONT.5, $934( \pm 28 \mathrm{pg} / \mathrm{ml})$ are compared to 463 control normoxic CdM $225( \pm 24 \mathrm{pg} / \mathrm{ml})$ as shown in (Figure 7).

464

465 466

\section{Effect of Conditioned-Medium (CdM) on Migration of HUVECs}

The role of the CdM in promoting migration of HUVECs was investigated using migration assay to confirm whether secretions of $\mathrm{CSCs}^{\mathrm{HYP}}$ enhance angiogenesis in the tumor microenvironment. HUVECs were tested for their ability to migrate toward CdM from different hypoxic episodes compared to their migration toward endothelial basal medium (EBM-2) serum and supplement free, as a control, using transwell migration assay. The Transwell migration assay is used to create a chemical gradient by putting the $\mathrm{CdM}$ in the lower chamber. Comparing the migration of HUVECs from upper chamber across the membrane upon using the EBM-2 without serum and without supplements (control) group versus using the CdM obtained from hypoxic shots, it was results showed that there were more purple-stained cells migrating toward the CdM obtained from the CONT.5 shot than the other two episodes (CONT.10 and CONT.15). The CdM obtained from the INTR.20 episode had also more purple-stained cells migrating towards it, compared to the other three episodes (INTR.10, INTR.30, and INTR.40). Interestingly, a significant difference found was that the migrated-cell count in HUVECs towards CdM from vs control was $38000( \pm 2.6)$ vs. $4500( \pm 1.5)$. These findings suggest that the secretions presented in the CdM which were obtained from CONT.5 and INTR.20 episodes significantly promoted the migration of HUVECs. The data also showed that the migration capacity of HUVECs increased significantly (10-fold) when cultured using the CdM from CONT.5 shot and (8.4-fold) when cultured using the CdM from INTR.20 shot compared with HUVECs cultured with control (Figure 8). To clarify the interaction between endothelial cells and $\mathrm{CSCs}^{\mathrm{HYP}}$ in the tumor microenvironment, HUVECs were examined in direct and indirect co-cultures with the $\mathrm{CSCs}^{\mathrm{HYP}}$ subpopulation that underwent CONT.5 episode. The control of HUVECs was cultured in serum and supplement free media and possessed a 'teardrop-like' morphology. In the direct co-culture model, the 
494 endothelial cell morphology changed and acquired elongated threadlike shape as a response to

495 the influence of $\mathrm{CSCs}^{\mathrm{HYP}}$ secretions though the growth media was free from serum and any

496 growth factors as shown in (Figure 9. A, B, C).

497 We also examined the growth of HUVECs indirectly co-cultured with CSCs ${ }^{\mathrm{HYP}}$ using a $0.4 \mathrm{uM}$

498 permeable membrane that was placed between HUVECs and $\mathrm{CSCs}^{\mathrm{HYP}}$ subpopulation. Indirect

499 co-culture of the subpopulation of CONT.5 CSCs ${ }^{\mathrm{HYP}}$ with HUVECs induced sequential

500 morphological changes. As a response to the influence of secretions from $\mathrm{CSCs}^{\mathrm{HYP}}$ thattrans-

501 passed through the membrane, the morphology of HUVECs began to change at 72 hours,

502 forming net-like structures resembling a vascular network, though the growth media was free

503 from serum and any growth factors (Figure 9-D \& E). Control HUVECs proliferation reached a

504 plateau in 24 hours after which cells started to die.

505

506 In Vitro Wound-Healing Assay of HUVECs

507 This assay was done to assess how secretions from $\mathrm{CSCs}^{\mathrm{HYP}}$ in the tumor microenvironment

508 would affect the ability of HUVECs to migrate in response to chemoattractants in the CdM

509 obtained from different hypoxic shots. This model mimics the tumor microenvironment where

510 angiogenic ability of endothelial cells is affected by secretions from $\mathrm{CSCs}^{\mathrm{HYP}}$. In the control

511 experiment, HUVECs were treated with CdM obtained from $\mathrm{CSCs}^{\mathrm{NOR}}$. In the presence of the

512 CdM obtained from hypoxic shots, HUVECs were able to migrate and the wound closure was

513 enhanced after 48 hours. The wound closure was significantly enhanced by CdM obtained from

514 INTR.20 (Figure 10-B) and CONT.5 (Figure 10-C) shots. Whereas in the case of the control,

515 HUVECs that were treated with CdM media obtained from $\mathrm{CSCs}^{\mathrm{NOR}}$, showed a weaker capacity

516 to migrate. The wound surface area could not be calculated in order to assess wound closure

517 because the HUVECs treated with $\mathrm{CdM}$ obtained from $\mathrm{CSCs}^{\mathrm{NOR}}$ and from $\mathrm{CSCs}^{\mathrm{HYP}}$ after long-

518 term exposure (INTR.40 and CONT.15) started to die after 16 hours of treatment which

519 prevented further quantitative measurements.

520

521 In Vitro Wound-Healing Assay of Hypoxic CSCs

522 Due to the significant role that CSCs play in tumor recurrence and metastasis, a wound-healing

523 assay was performed. CSCs were seeded as a (2D) monolayer, scratched, and then cell migration

524 was evaluated. The seeded CSCs were originally expanded and grown in hypoxic conditions,

525 INTR.10, INTR.20, INTR.30, INTR.40, CONT.5, CONT.10, and CONT.15 in addition to the

526 control CSCs in normoxia. The scratched $\mathrm{CSCs}^{\mathrm{HYP}}$ and $\mathrm{CSCs}^{\mathrm{NOR}}$ cultured in DMEM/F-12

527 without serum were assessed for wound closure ability which was determined by calculating the 
528 ratio of the wound surface area at the endpoint (36 hours) to its surface area at the starting point

529 of the experiment (zero hour). Images were analyzed by ImageJ software. Subpopulations of $530 \mathrm{CSCs}^{\mathrm{HYP}}$ showed greater ability for wound closure compared to $\mathrm{CSCs}^{\mathrm{NOR}}$ (Figure 11-12).

$531 \mathrm{CSCs}^{\mathrm{HYP}}$ from INTR.20 and from CONT.5 manifested the greatest wound closure (Figure 11).

532 At 36 hours, the wound closure of the control was only $40 \%$, while it was $98 \%$ and $95 \%$ for

533 INTR.20 (Figure 12-C) and CONT.5 (Figure 12-F), respectively.

534

535 DISCUSSION

536 The initial stage of our study focused on the formation and characterization of MCF-7 537 mammospheres enriched with CSCs. The mammosphere culture is an approach to culturing 538 cancer cells in a three-dimensional (3D) conformation in vitro. These 3D models have been well 539 recognized in breast cancer research due to the great similarity of these models to the in vivo 540 solid tumors (Weiswald, Bellet et al. 2015, Boo, Ho et al. 2016). The 3D models are not only 541 biologically spherical in shape, but also possess many shared features with solid tumors which 542 were not observed in traditional 2D monolayer cultures (Baker and Chen 2012, Ekert, Johnson et 543 al. 2014). Interestingly, several current reports have shown that CSCs are particularly enriched 544 and maintained in cultures of 3D mammospheres (López, Poitevin et al. 2012, Liu, Ma et al. 545 2013). CSCs show a tendency to differentiate in the availability of serum, accordingly serum546 free culture conditions are believed to maintain the CSCs in an undifferentiated stage supporting 547 their enrichment, with recognizable loss and reduction in the size of the upper cell in the 548 hierarchical structure (Kruyt \& Schuringa 2010; Lin et al. 2012). The results of our study have 549 shown a CD44+/CD24- expression (Table 1) for parental MCF-7 of (1\%). After being cultured 550 in a low-adherence, serum-free, with supplements media, CSCs were sorted and again identified 551 with (FACS) and showed an extreme elevation of CD44+/CD24- expression to (81.3\%) only 552 three days after being grown in mammosphere culture conditions, while it was (33.2\%) before 553 being sorted as CSCs in mammosphere culture media. Interestingly, it reverted to (35.5\%) at day 554 21. From these results, it is concluded that sorting of CSCs allows them to acquire stemness 555 character that is much higher than their parental cells and their unsorted population, and declines 556 over time. The interpretation is that CSCs upon repetitive and continuous growth are driven 557 towards differentiation into regular cancer cells instead of bearing a CSC state. In another 558 interpretation, a distinctive characteristic of mammosphere culture conditions -unlike the 559 adherent cell culture- is that during the passaging of CSCs most of them die early (through 560 apoptosis/anoikis) and the mitogen-responsive anoikis resistant cells that were supposed to be 561 CSCs grow and form new mammospheres. Therefore, increased expression of $\mathrm{CD}_{4} 4^{+} / \mathrm{CD}^{2} 4^{-}$ 
562 within mammospheres compared to parent cells could be due to an enriched anoikis-resistant 563 cancer stem cells proliferation process (Deleyrolle et al. 2011).

564 The second stage of our study was to investigate the role of cycling hypoxia on CSCs 565 propagation, stemness character, drug resistance, and adequate motility to recruit new tumor 566 growth at distant sites through migration and induction of VEGF expression level. In our study 567 as detailed in methods, we divided CSCs into two groups to be exposed to intermittent or 568 continuous shots of hypoxia. MTT cytotoxicity assay was performed to assess chemoresistance 569 of CSCs in hypoxia vs. normoxia vs. parent MCF-7 cells using doxorubicin. Doxorubicin is a 570 cytotoxic anthracycline, that is widely used drug to treat breast cancer. The cytotoxic effect of 571 doxorubicin on malignant cells is due to nucleotide base intercalation and cell membrane lipid 572 binding. Intercalation inhibits nucleotide replication and action of DNA and RNA polymerases. 573 The interaction of doxorubicin with topoisomerase II to form DNA-cleavable complexes appears 574 to be an important mechanism of doxorubicin cytocidal activity (Miglietta et al. 2000). Our 575 results have shown that parent $\mathrm{MCF}-7$ had the lowest drug resistance to doxorubicin $576\left(\mathrm{IC}_{50}=0.46 \mathrm{uM}\right)$ compared to normoxic CSCs mammosphere $\left(\mathrm{IC}_{50}=1.90 \mathrm{uM}\right)$, and when both 577 were compared to $\mathrm{CSCs}^{\mathrm{HYP}}$ that underwent hypoxic episodes of INTR.20 and CONT.5, they had $578\left(\mathrm{IC}_{50}=6.20 \mathrm{uM}\right)$ and $\left(\mathrm{IC}_{50}=7.12 \mathrm{uM}\right)$, respectively. Consequently, one concludes that hypoxia 579 remarkably elevates chemoresistance not only upon comparison with parent MCF-7, but also 580 upon comparison with the $\mathrm{CSCs}^{\mathrm{NOR}}$ (control). It is noteworthy to explain that the $\mathrm{IC}_{50}$ values of $581 \mathrm{CSCs}^{\mathrm{HYP}}$ subjected to all these hypoxic episodes (INTR.10, INTR.20, INTR.30, CONT.5, and 582 CONT.10) were higher than parental MCF-7 and than CSCs ${ }^{\mathrm{NOR}}$ (control), with an exception for 583 the $\mathrm{CSCs}^{\mathrm{HYP}}$ that underwent (INTR.40 and CONT.15) episodes representing the finale of the 584 hypoxic treatment which had $\mathrm{IC}_{50}$ values that were less than the $\mathrm{IC}_{50}$ of $\mathrm{CSCs}^{\mathrm{NOR}}$ (control) and 585 close to parental MCF-7. It is notable to compare MTT assay results with flow cytometry results 586 of the $\mathrm{CSCs}^{\mathrm{HYP}}$ as they reflect one another. MTT assay results for sorted CSCs have shown 587 higher chemoresistance with (4-fold) increased $\mathrm{IC}_{50}$ compared to parental MCF-7 whose $588 \mathrm{CD} 44^{+} / \mathrm{CD} 24^{-}$expression was $(1 \%)$. This proposes the likelihood that increased stemness is the 589 contributor to the higher drug resistance in mammospheres as suggested previously (Calcagno et 590 al. 2010). Moreover, identification of $\mathrm{CD} 44^{+} / \mathrm{CD} 24^{-}$expression by flow cytometry applied on $591 \mathrm{CSCs}^{\mathrm{HYP}}$ that underwent CONT.5 and INTR.20 revealed their possession of the highest 592 expression at $51.6 \%$ and $39.8 \%$, respectively. Among all hypoxic episodes, the lowest 593 expressions of $0.5 \%$ and $0.3 \%$ were for $\mathrm{CSCs}^{\mathrm{HYP}}$ that underwent CONT.15 and INTR.40, 594 respectively. Interestingly, $\mathrm{CSCs}^{\mathrm{HYP}}$ that underwent CONT.5 and INTR.20, which had the 
595 highest $\mathrm{CD} 44^{+} / \mathrm{CD} 24^{-}$expression also had the highest chemoresistance results in contrast to

596 CONT.15 and INTR.40 which had the lowest CD44 ${ }^{+} / \mathrm{CD} 24^{-}$expression, and also the lowest

597 chemoresistance results. These results agree with their counterparts in past studies which showed

598 that chemoresistance is closely related to many fundamental or acquired characteristics of CSCs,

599 such as DNA repair ability, quiescence, overexpression of antiapoptotic enzymes, drug efflux

600 transporters and detoxifying enzymes (Vinogradov \& Wei 2012). Our findings also agree with

601 the study of (Hermann et al. 2007) that reported the existence of a subpopulation of pancreatic

602 cancer cells which resembled stem cells and had higher gemcitabine resistance both in vitro and

603 in vivo. Since CSCs play a significant role in tumor recurrence and metastasis, wound-healing

604 was investigated to evaluate the behavior of CSCs after being exposed to different hypoxic shots.

605 The adherent $\mathrm{CSCs}^{\mathrm{HYP}}$ showed greater ability of self-renewal and wound healing, especially after

606 being exposed to INTR.20 and CONT.5 hypoxic shots. They also showed the slowestwound

607 healing rate after being exposed to INTR.40 and CONT.15. The wound healing results were

608 mirror-image reflection of the chemoresistance and stemness characteristics results relevant to

609 each hypoxic episode discussed above.

610 The third phase of this study was performed to evaluate the role of secretions of CSCs after

611 being subjected to different hypoxic shots on angiogenesis using (HUVECs) as a model

612 proposed to modulate neovascularization phenotype. Increased VEGF secretion in $\mathrm{CSCs}^{\mathrm{HYP}}$

613 versus control $\mathrm{CSCs}^{\mathrm{NOR}}$ was confirmed. This finding demonstrated that $\mathrm{CSCs}^{\mathrm{HYP}}$ secretions

614 increased the level of VEGF significantly which is linked to the angiogenic potential of the CdM

615 reported in this study. In this manner, the study of (Bao et al. 2006) showed that CD133+

616 enriched stem cell-like glioma cells (SCLGC) produced tumors with an increased tumor

617 vascularity, necrosis, and hemorrhage. Also, the authors showed that the VEGF expression was

618 10-20 fold up-regulated in $\mathrm{CD}_{133^{+}}$SCLGC.

619 The direct and indirect co-culture of $\mathrm{CSCs}^{\mathrm{HYP}}$ that underwent CONT.5 with endothelial 620 HUVECs proved that this co-culture system comprised constituents that promoted angiogenic 621 phenotype, such as proliferation and differentiation of endothelial cell into a net-like structure.

622 Interestingly, the influence of the secretions from $\mathrm{CSCs}^{\mathrm{HYP}}$ modified the HUVECs morphology

623 to a longer, mesenchymal-like appearance and enhanced their migration rate. The migration 624 assay confirmed that $\mathrm{CdM}$ obtained from $\mathrm{CSCs}^{\mathrm{HYP}}$ enhanced the migration rate of HUVECs 625 compared to its control group cultured in EBM-2. HUVECs migrated towards the 626 chemoattractants that $\mathrm{CSCs}^{\mathrm{HYP}}$ secreted in the $\mathrm{CdM}$ that were obtained from all hypoxic 627 episodes, except the CdM that were obtained from CONT.15 and INTR.40, which did not 
628 enhance HUVECs migration. However, migration was distinctive upon the use of CdM obtained

629 from CONT.5 and INTR.20. These results could be interpreted in light of the biological process

630 in which the growth of a new blood vessel from pre-existing blood cells via "sprouting" of

631 endothelial cells is led by a tip cell which is a single endothelial one. Tip cells drive vascular

632 growth by detecting gradient proangiogenic mediators such as VEGF. Adjacent endothelial cells

633 become stalk cells that can proliferate and migrate towards the tip cell which results in the

634 elongation of the sprouting vessel (Gerhardt et al. 2003).

635

636 The wound healing assay has confirmed that the CdM obtained from CSCs ${ }^{\mathrm{HYP}}$ enhanced the

637 repair of the scratch in HUVECs, and closure was significantly higher upon the use of CdM

638 obtained from CONT.5 and INTR.20 episodes in comparison to wound closure treated with

$639 \mathrm{CdM}$ media obtained from $\mathrm{CSCs}^{\mathrm{NOR}}$. Moreover, the addition of CdM obtained from $\mathrm{CSCs}^{\mathrm{HYP}}$

640 cultures into HUVECs was also found to promote the endothelial cell proliferation and induce

641 capillary-like tube structure formation by increasing total length of the formed branched-tube,

642 the number of loops and the area covered by the tubes. This observation was distinctive in the

643 use of $\mathrm{CdM}$ obtained from $\mathrm{CSCs}^{\mathrm{HYP}}$ exposed to CONT.5 and to INTR.20. Together, these

644 results suggest that $\mathrm{CSCs}^{\mathrm{HYP}}$ cells produce pro-angiogenic factors that may directly alter the

645 behavior of endothelial cells. Such findings are in agreement with the study of (Tu et al. 2009)

646 which showed that A549 lung cancer cells hypoxic CdM improved HUVECs' cell presence and

647 wound healing migration ability.

648

649 In conclusion, the angiogenic components and the angiogenic switch were enhanced by the

650 tumor microenvironment constructed by the $\mathrm{CdM}$ of $\mathrm{CSCs}^{\mathrm{HYP}}$ particularly after 5 hypoxic

651 episodes of continuous 72-hours weekly exposure (CONT.5) and 20 episodes of intermittent 8-

652 hours exposure to hypoxia, 3 times weekly (INTR.20).

653

654 In the context of the above-detailed findings and upon comparing the results of CSCs exposed to

655 intermittent versus continuous hypoxic condition in terms of stemness, chemoresistance, tube

656 formation and VEGF protein expression related to angiogenesis, wound healing and finally, cell

657 migration, the results implied similarity among intermittent and continuous treatments between

658 the early intervals (INTR.20 and CONT.5), between the intermediate intervals (INTR.30 and

659 CONT.10), and also between the late intervals (INTR.40 and CONT.15).

660 The present study agrees with the study of (Liu et al. 2017) which demonstrated that

661 intermittent and continuous hypoxic conditions significantly increased the migration of

662 MDA-MB-231 breast cancer cells to a certain extent, either because of excessive long-term 
663 exposure or because of the increased number of hypoxia-reoxygenation cycles. In this study, the 664 maximum increase of these features by hypoxic effect was observed at early intervals (INTR.20 665 and CONT.5), whereas it started to decline at intermediate ones (INTR.30 and CONT.10), and 666 drastically declined at late intervals (INTR.40 and CONT.15) to a rate even lower than the 667 control group of CSCs in normoxia

668 The previous study demonstrated that excessive long-term or high-frequency exposure to 669 hypoxia leads to the generation of reactive oxygen species (ROS), which in turn, induces lipid 670 peroxidation and increases the production of stress responding proteins, thus promotes DNA 671 strand breakage, cellular injury, and apoptosis (Pires et al. 2010; Zepeda et al. 2013). Generally, 672 low levels of ROS are required for stem cells to maintain quiescence and self-renewal. 673 Therefore, in the CSCs scenario, a low concentration of ROS enhances their stemness and 674 contributes to tumorigenesis (Liu \& Wang 2015). However, increased ROS production due to 675 extensive hypoxia causes CSCs exhaustion, differentiation, senescence, and apoptosis (Zhou et 676 al. 2014).

677 Thus, it is interpreted that the ROS level began to increase starting from the intermediate 678 intervals (INTR.30 and CONT.10) and reached maximal rate in the late intervals (INTR.40 and 679 CONT.15) proposing the manifestation of differentiation, senescence, and apoptosis in CSCs 680 extensively exposed to hypoxia, based on the previous explanation.

681

682

\section{CONCLUSION}

684

685 This study has proven that the highly optimized hypoxia-reoxygenation system applied to CSCs 686 has enriched their growth, enhanced their properties of self-renewal, increased the expression of 687 stemness surface markers, and increased their chemoresistance. The findings of this study also 688 demonstrated that the $\mathrm{CSCs}^{\mathrm{HYP}}$ microenvironment plays a major role in enhancing angiogenesis 689 and tumor vascularization by recruiting endothelial cells into the tumor microenvironment, and 690 in activating distinct molecular mechanisms that need further investigations. Whether the 691 elevation of stemness in $\mathrm{CSCs}^{\mathrm{HYP}}$ results from the de-differentiation of differentiated MCF-7 692 cells, or from the enhanced proliferation of CSCs after hypoxic exposure is still unclear and 693 needs further studies. The interactions between CSCs in tumor microenvironment and other 694 contributing processes such as hypoxia and angiogenesis are still at the infancy stage and need 695 in-depth future research.

696 


\section{REFERENCES}

698 Al-Hajj M, Wicha MS, Benito-Hernandez A, Morrison SJ, and Clarke MF. 2003. Prospective

699

700

701

702

703

704

705

706

707

708

709

710

711

712

713

714

715

716

717

718

719

720

721

722

723

724

725

726

727

728

729

730

731

732

733

734

735 identification of tumorigenic breast cancer cells. Proceedings of the National Academy of Sciences 100:3983-3988.

Bao S, Wu Q, Sathornsumetee S, Hao Y, Li Z, Hjelmeland AB, Shi Q, McLendon RE, Bigner DD, and Rich JN. 2006. Stem cell-like glioma cells promote tumor angiogenesis through vascular endothelial growth factor. Cancer research 66:7843-7848.

Batlle E, and Clevers H. 2017. Cancer stem cells revisited. Nature medicine 23:1124.

Bristow RG, and Hill RP. 2008. Hypoxia and metabolism: hypoxia, DNA repair and genetic instability. Nature Reviews Cancer 8:180.

Brurberg KG, Graff BA, Olsen DR, and Rofstad EK. 2004. Tumor-line specific pO2 fluctuations in human melanoma xenografts. International Journal of Radiation Oncology• Biology• Physics 58:403-409.

Calcagno AM, Salcido CD, Gillet J-P, Wu C-P, Fostel JM, Mumau MD, Gottesman MM, Varticovski L, and Ambudkar SV. 2010. Prolonged drug selection of breast cancer cells and enrichment of cancer stem cell characteristics. Journal of the National Cancer Institute 102:1637-1652.

Cassavaugh J, and Lounsbury KM. 2011. Hypoxia-mediated biological control. Journal of cellular biochemistry 112:735-744.

Chaplin DJ, Olive PL, and Durand RE. 1987. Intermittent blood flow in a murine tumor: radiobiological effects. Cancer research 47:597-601.

Chenevier-Gobeaux C, Simonneau C, Lemarechal H, Bonnefont-Rousselot D, Poiraudeau S, Rannou F, Ekindjian O, Anract P, and Borderie D. 2013. Effect of hypoxia/reoxygenation on the cytokine-induced production of nitric oxide and superoxide anion in cultured osteoarthritic synoviocytes. Osteoarthritis and cartilage 21:874-881.

Crowder SW, Balikov DA, Hwang Y-S, and Sung H-J. 2014. Cancer stem cells under hypoxia as a chemoresistance factor in the breast and brain. Current pathobiology reports 2:33-40.

Deleyrolle LP, Ericksson G, Morrison BJ, Lopez JA, Burrage K, Burrage P, Vescovi A, Rietze RL, and Reynolds BA. 2011. Determination of somatic and cancer stem cell selfrenewing symmetric division rate using sphere assays. PloS one 6:e15844.

Denko NC. 2008. Hypoxia, HIF1 and glucose metabolism in the solid tumour. Nature Reviews Cancer 8:705.

Eales K, Hollinshead K, and Tennant D. 2016. Hypoxia and metabolic adaptation of cancer cells. Oncogenesis 5:e190.

Gerhardt H, Golding M, Fruttiger M, Ruhrberg C, Lundkvist A, Abramsson A, Jeltsch M, Mitchell C, Alitalo K, and Shima D. 2003. VEGF guides angiogenic sprouting utilizing endothelial tip cell filopodia. The Journal of cell biology 161:1163-1177.

Gordan JD, Lal P, Dondeti VR, Letrero R, Parekh KN, Oquendo CE, Greenberg RA, Flaherty KT, Rathmell WK, and Keith B. 2008. HIF- $\alpha$ effects on c-Myc distinguish two subtypes 
736

737

738

739

740

741

742

743

744

745

746

747

748

749

750

751

752

753

754

755

756

757

758

759

760

761

762

763

764

765

766

767

768

769

770

771

772

of sporadic VHL-deficient clear cell renal carcinoma. Cancer cell 14:435-446.

Hammer S, To KK, Yoo Y-G, Koshiji M, and Huang LE. 2007. Hypoxic suppression of the cell cycle gene CDC25A in tumor cells. Cell Cycle 6:1919-1926.

Hermann PC, Huber SL, Herrler T, Aicher A, Ellwart JW, Guba M, Bruns CJ, and Heeschen C. 2007. Distinct populations of cancer stem cells determine tumor growth and metastatic activity in human pancreatic cancer. Cell stem cell 1:313-323.

Huang R, and Rofstad EK. 2017. Cancer stem cells (CSCs), cervical CSCs and targeted therapies. Oncotarget 8:35351.

Kaur B, Khwaja FW, Severson EA, Matheny SL, Brat DJ, and Van Meir EG. 2005. Hypoxia and the hypoxia-inducible-factor pathway in glioma growth and angiogenesis. Neurooncology 7:134-153.

Kerbel RS. 2008. Tumor angiogenesis. New England Journal of Medicine 358:2039-2049.

Kietzmann T, Mennerich D, and Dimova EY. 2016. Hypoxia-inducible factors (HIFs) and phosphorylation: impact on stability, localization, and transactivity. Frontiers in cell and developmental biology 4:11.

Kong W, He L, Richards E, Challa S, Xu C, Permuth-Wey J, Lancaster J, Coppola D, Sellers T, and Djeu J. 2014. Upregulation of miRNA-155 promotes tumour angiogenesis by targeting VHL and is associated with poor prognosis and triple-negative breast cancer. Oncogene 33:679.

Kruyt FA, and Schuringa JJ. 2010. Apoptosis and cancer stem cells: Implications for apoptosis targeted therapy. Biochemical pharmacology 80:423-430.

Lin S-P, Lee Y-T, Wang J-Y, Miller SA, Chiou S-H, Hung M-C, and Hung S-C. 2012. Survival of cancer stem cells under hypoxia and serum depletion via decrease in PP2A activity and activation of p38-MAPKAPK2-Hsp27. PloS one 7:e49605.

Liu J, and Wang Z. 2015. Increased oxidative stress as a selective anticancer therapy. Oxidative medicine and cellular longevity 2015.

Liu L, Liu W, Wang L, Zhu T, Zhong J, and Xie N. 2017. Hypoxia-inducible factor 1 mediates intermittent hypoxia-induced migration of human breast cancer MDA-MB-231 cells. Oncology letters 14:7715-7722.

Mellor H, Snelling S, Hall M, Modok S, Jaffar M, Hambley T, and Callaghan R. 2005. The influence of tumour microenvironmental factors on the efficacy of cisplatin and novel platinum (IV) complexes. Biochemical pharmacology 70:1137-1146.

Miglietta A, Cavalli R, Bocca C, Gabriel L, and Gasco MR. 2000. Cellular uptake and cytotoxicity of solid lipid nanospheres (SLN) incorporating doxorubicin or paclitaxel. International journal of pharmaceutics 210:61-67.

Muz B, de la Puente P, Azab F, and Azab AK. 2015. The role of hypoxia in cancer progression, angiogenesis, metastasis, and resistance to therapy. Hypoxia 3:83. 
773

774

775

776

777

778

779

780

781

782

783

784

785

786

787

788

789

790

791

792

793

794

795

796

797

798

799

800

801

802

803

804

Ojha T, Pathak V, Shi Y, Hennink WE, Moonen CT, Storm G, Kiessling F, and Lammers T. 2017. Pharmacological and physical vessel modulation strategies to improve EPRmediated drug targeting to tumors. Advanced drug delivery reviews 119:44-60.

Peitzsch C, Tyutyunnykova A, Pantel K, and Dubrovska A. 2017. Cancer stem cells: the root of tumor recurrence and metastases. Semin Cancer Biol: Elsevier. p 10-24.

Ping YF, and Bian XW. 2011. Concise review: contribution of cancer stem cells to neovascularization. Stem Cells 29:888-894.

Pires IM, Bencokova Z, Milani M, Folkes LK, Li J-L, Stratford MR, Harris AL, and Hammond EM. 2010. Effects of acute versus chronic hypoxia on DNA damage responses and genomic instability. Cancer research:0008-5472. CAN-0009-2715.

Senthebane DA, Rowe A, Thomford NE, Shipanga H, Munro D, Al Mazeedi MA, Almazyadi HA, Kallmeyer K, Dandara C, and Pepper MS. 2017. The role of tumor microenvironment in chemoresistance: To survive, keep your enemies closer. International journal of molecular sciences 18:1586.

Tu ML, Wang HQ, Chen LJ, Lu JC, Jiang F, Liang JH, and Li DS. 2009. Involvement of Akt1/protein kinase $\mathrm{B} \alpha$ in tumor conditioned medium-induced endothelial cell migration and survival in vitro. Journal of cancer research and clinical oncology 135:1543-1550.

Vinogradov S, and Wei X. 2012. Cancer stem cells and drug resistance: the potential of nanomedicine. Nanomedicine 7:597-615.

Wang L, Zhang L, Shen W, Liu Y, and Luo Y. 2016. High expression of VEGF and PI3K in glioma stem cells provides new criteria for the grading of gliomas. Experimental and therapeutic medicine 11:571-576.

Wang Z, Butner JD, Kerketta R, Cristini V, and Deisboeck TS. 2015. Simulating cancer growth with multiscale agent-based modeling. Seminars in cancer biology: Elsevier. p 70-78.

Zepeda AB, Pessoa Jr A, Castillo RL, Figueroa CA, Pulgar VM, and Farías JG. 2013. Cellular and molecular mechanisms in the hypoxic tissue: role of HIF-1 and ROS. Cell biochemistry and function 31:451-459.

Zhao Y, and Adjei AA. 2015. Targeting angiogenesis in cancer therapy: moving beyond vascular endothelial growth factor. The oncologist 20:660-673.

Zhou D, Shao L, and Spitz DR. 2014. Reactive oxygen species in normal and tumor stem cells. Advances in cancer research: Elsevier, 1-67. 
Figure 1

Summary of methodology workflow adopted in this project

The first stage was to establish CSCs sub-population from parental MCF-7 cells by several steps of culturing cells in ultra-low serum-free media and sorting to enrich stemness biomarker that was finally identified by flow cytometry after 21 days. Stage two included exposing CSCs to hypoxic and normoxic conditions in optimized intervals for over than four months and collecting conditioned starvation media in which they were grown. Also, multiple assays were performed using hypoxic and normoxic CSCs to test the hypoxia effect on CSCS. Stage three was performed to assess the effect of CSCs secretions in the conditionedmedium on angiogenesis.

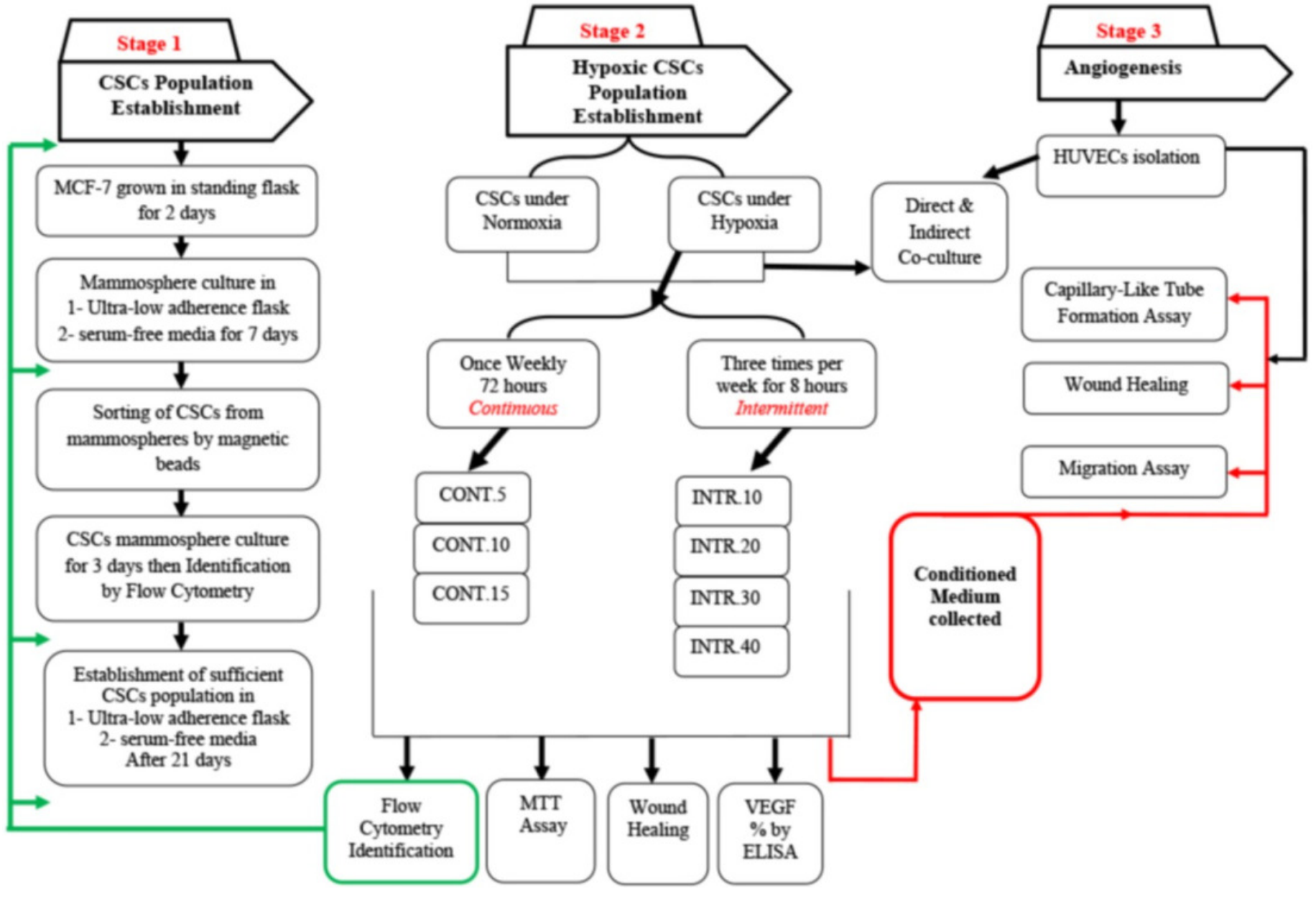




\section{Figure 2}

The morphology of MCF-7 mammospheres

(A) MCF-7 floating cells in serum-free low adherent conditions required for mammospheres

formation. (B) At Day-3 the (3D) mammospheres began to structure. (C) At Day-7 larger and denser (3D) mammospheres have structured in a rigid and compact form. Representative images magnification (10x objective) and 30 um scale bar by Olympus inverted microscope. 

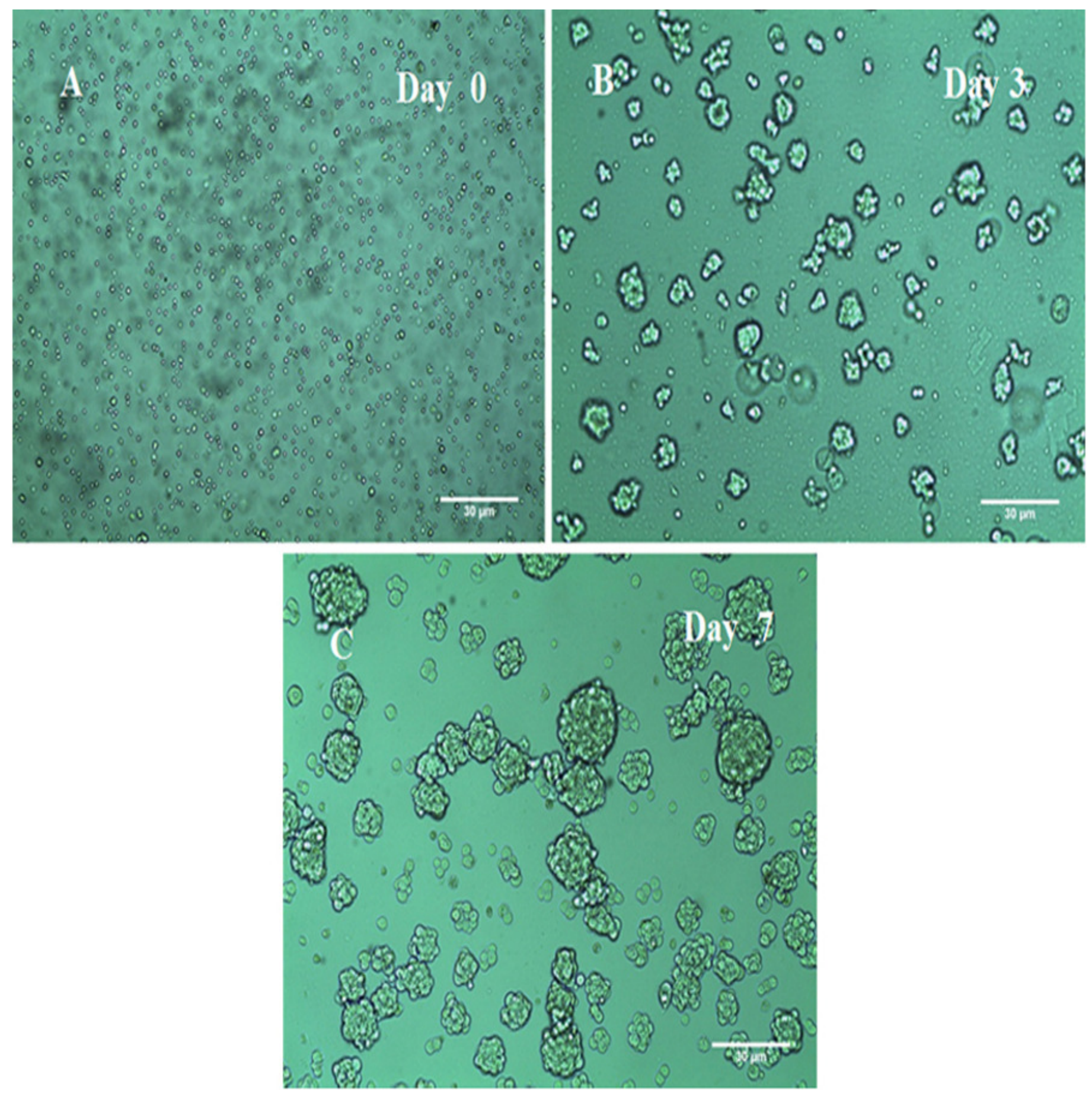


\section{Figure 3}

The 3D morphology of CSCs mammospheres under hypoxic condition (B, C, D, E) or in normoxia as in (A).

The (3D) morphology of CSCs clustered in multicellular spheroids in serum-free media. (A) CSCs under normoxia. (B) and (C) CSCs exposed to INTR.20 and INTR.40 hypoxic shots respectively. (D) and (E) CSCs exposed to CONT.5 and CONT.15 hypoxic shots respectively. $\mathrm{CSCS}^{\mathrm{HYP}}$ that underwent CONT.5 and INTR.20 were denser and larger in number than $\mathrm{CSCS}^{\text {NOR. }}$. CSCS ${ }^{\mathrm{HYP}}$ that underwent CONT.15 and INTR.40 had the least growth compared to other hypoxic exposures and CSCs ${ }^{\mathrm{NOR}}$. Representative images magnification (10x objective) and 30 um scale bar by Olympus inverted microscope. 


Figure 4

Determination of the cancer stem cell-like properties in mammosphere forming cells based on surface marker $\mathrm{CD} 44^{+} / \mathrm{CD} 24$

(A) parent MCF-7 cells. (B) unsorted mammospheres. (C) sorted CSCs mammospheres after 3 days of sorting. (D) CSCs mammospheres after 21 days of sorting. (E) CSCs ${ }^{\mathrm{HYP}}$ underwent INTR.20 shots. (F) CSCS ${ }^{\mathrm{HYP}}$ underwent CONT.5 shots. (G) CSCs ${ }^{\mathrm{HYP}}$ underwent INTR.40 shots. (H) $\mathrm{CSCS}^{\mathrm{HYP}}$ underwent CONT.15 shots.

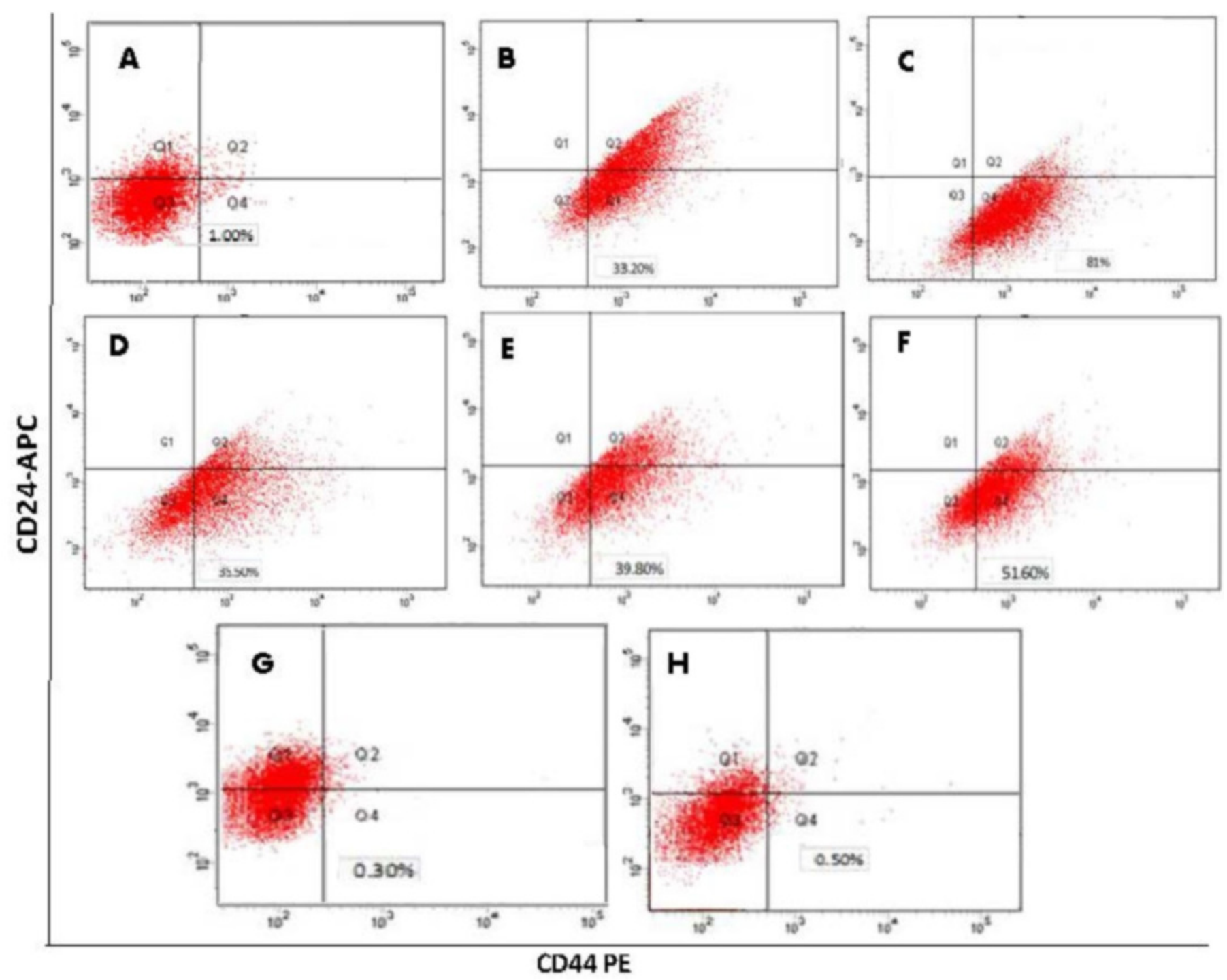




\section{Figure 5}

Effect of normoxic and hypoxic CdM on capillary-like tube structure formation in HUVECS.

HUVECs were cultured in CdM obtained from $\mathrm{CSCs}^{\mathrm{HYP}}$ mammospheres that were previously

grown and exposed to (B) CONT.5 (C) CONT.10 (D) CONT.15 (E) INTR.10 (F) INTR.20

INTR.30 (H) INTR.40 hypoxic shots and compared to HUVECs cultured using CdM obtained from $\mathrm{CSCs}^{\mathrm{NOR}}$ as a control as shown in (A). Capillary-like tubular structures were the highest in using CdM obtained from CONT.5 and INTR.20 exposure. Representative images magnification (4x objective) Olympus inverted microscope

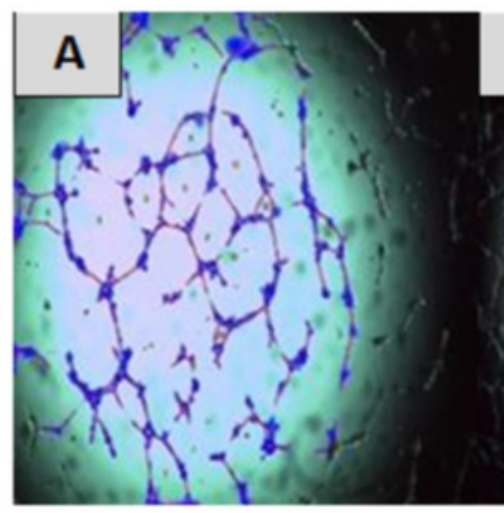

Control

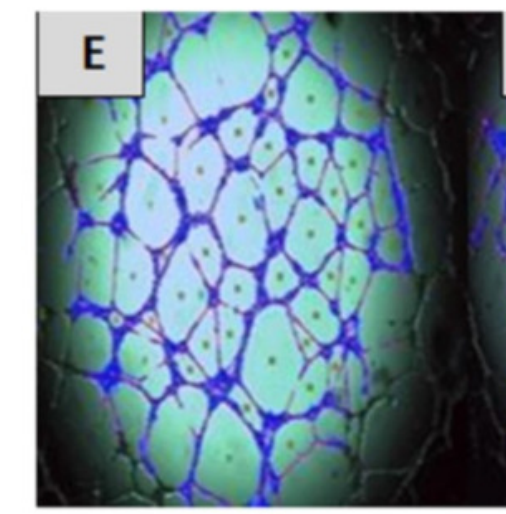

INTR.10

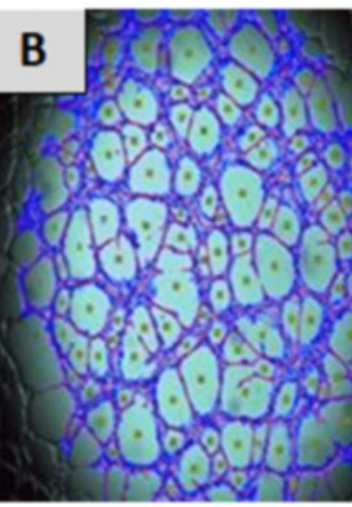

CONT.5

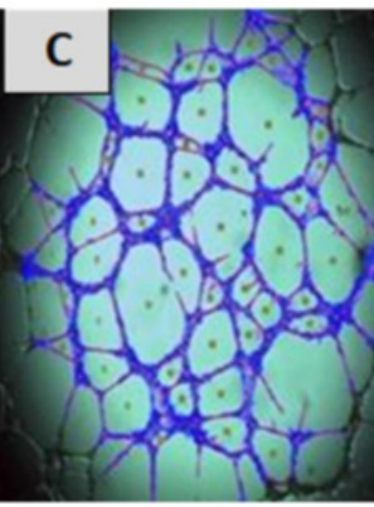

CONT.10

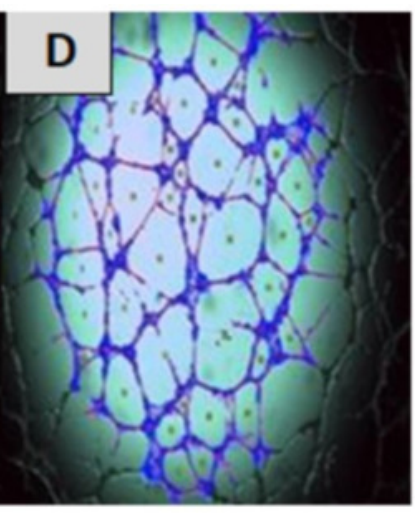

CONT.15

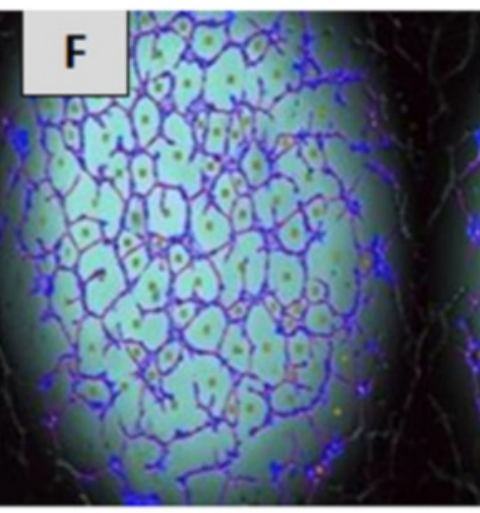

INTR.20

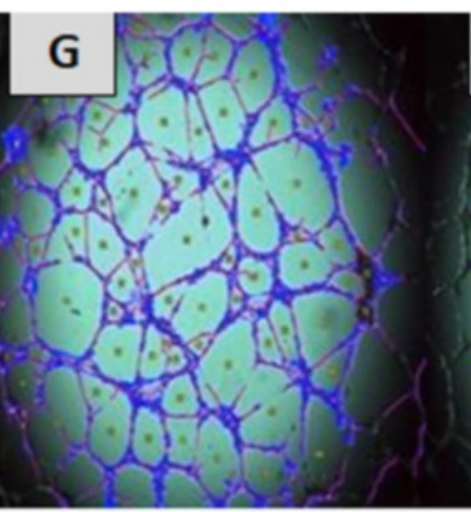

INTR.30

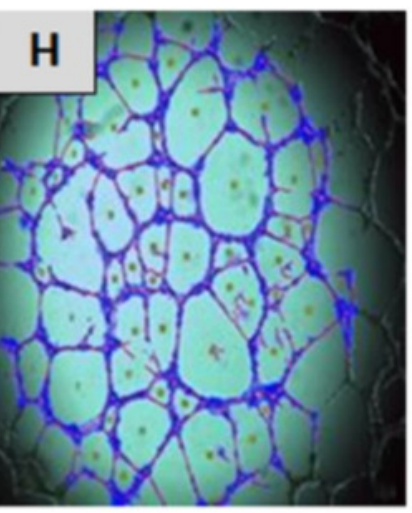

INTR.40 
Figure 6

Assessment of capillary-like tubular structure formation

(A) Total tube lengths formed. (B) Number of loops intersections. (C) Covered area

percentage. The results represent mean \pm SD of three independent experiments. The symbol marks the statistical significance levels as follows: $(*)$ indicates $p<(0.001)$ and $(* *)$ indicates $\mathrm{p}<(0.0001)$ as compared to the control.

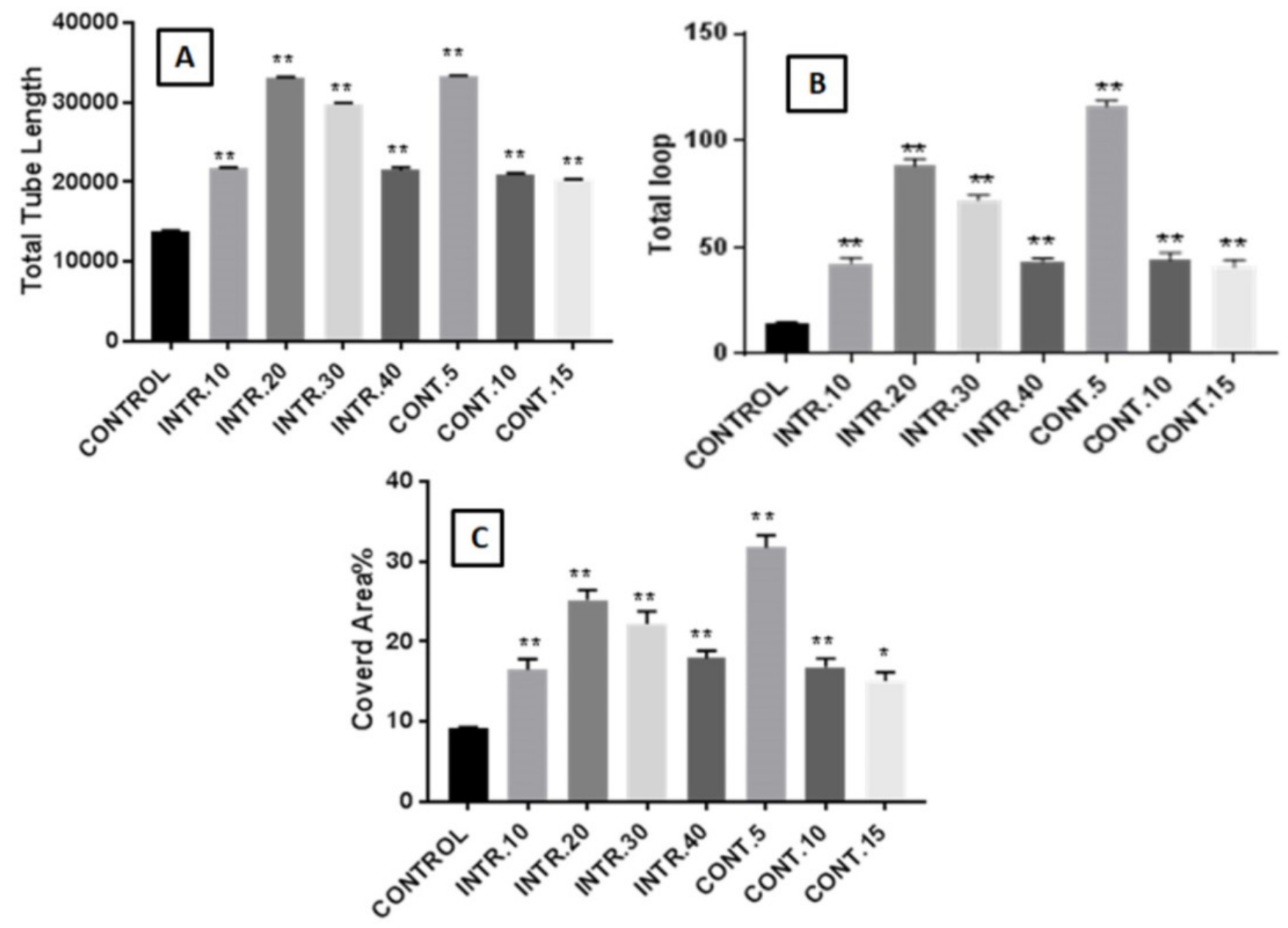


Figure 7

VEGF-A concentrations secreted by CSCs ${ }^{\mathrm{HPP}}$ or $\mathrm{CSCS}^{\mathrm{NOR}}$ were determined by ELISA assay

The results represent mean $\pm S D$ of three independent experiments. Statistically significant differences among the treated versus control cells are shown by $(*)\left(p^{<} 0.05\right)$ and $(* *)\left(p^{<}\right.$ $0.0001)$.

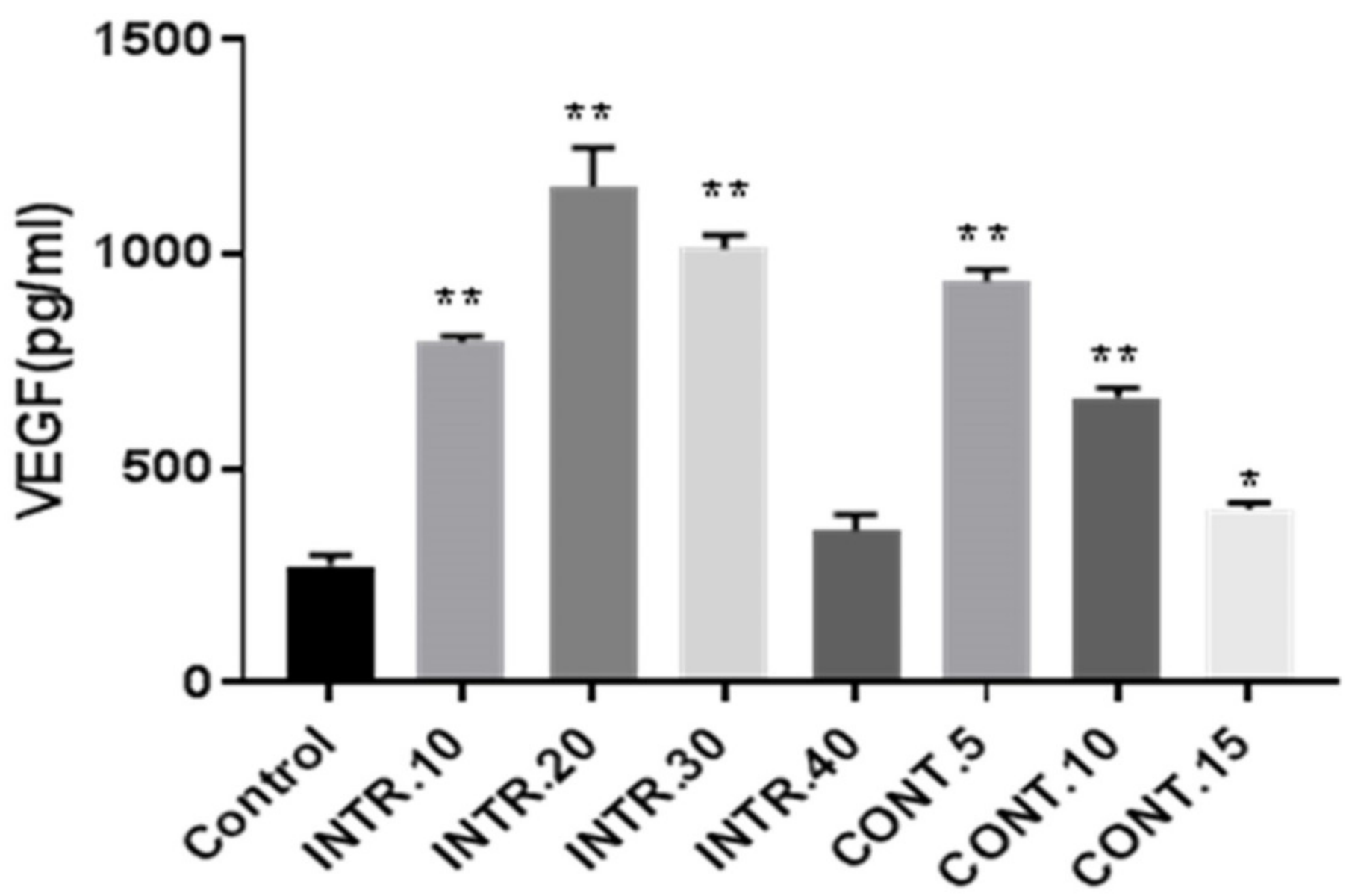


Figure 8

Transmigration of HUVECs induction by CdM obtained from CSCs ${ }^{\mathrm{HYP}}$ compared to control where EBM-2 serum-free served as control.

CdM from hypoxic shots CONT.5 and INTR.20 proved to be a potent chemoattractant compared to the control. All values were determined as averages of three independent experiments. The results represent mean $\pm S D$ of three independent experiments. Statistically significant differences among the treated and control cells are shown by $(* *)(p<0.0001)$.

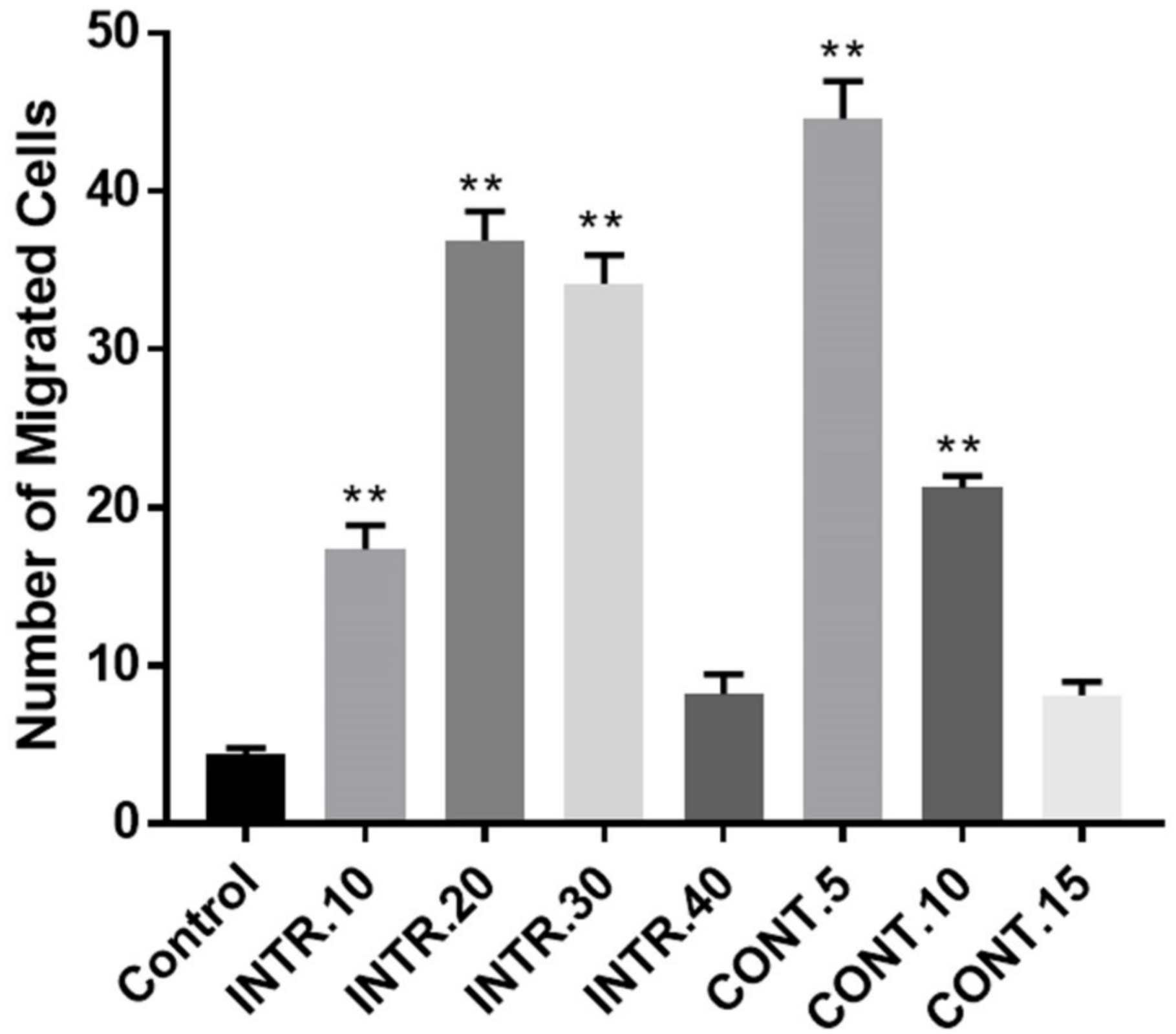




\section{Figure 9}

Morphological changes of HUVECs after co-cultured with CSCs ${ }^{\text {HYP }}$ subpopulation that underwent CONT.5

Hypoxic episode (A-C) for direct co-culture and (D-E) for indirect co-culture. Direct co-culture model: (A) CSCs ${ }^{\mathrm{HYP}}$ mammospheres were seeded directly above HUVECs monolayer at (0) time. (B) After 36 hours HUVECs proliferate normally with CSCs ${ }^{\mathrm{HYP}}$. (C) at 72 hours HUVECS obtained elongated threadlike shape mediated by $\mathrm{CSCS}^{\mathrm{HYP}}$ secretions. Indirect co-culture where permeable membrane was placed between HUVECs and CONT.5 CSCS $^{\text {HYP }}$ subpopulation: (D) HUVECs after 36 hours. (E) At 72 hours HUVECs morphology changed to form net-like structures resembling a vascular network as mediated by CSCs ${ }^{\text {HPP }}$ secretions that passed through the membrane. Representative images magnification ( $4 x$ objective) Olympus inverted microscope.

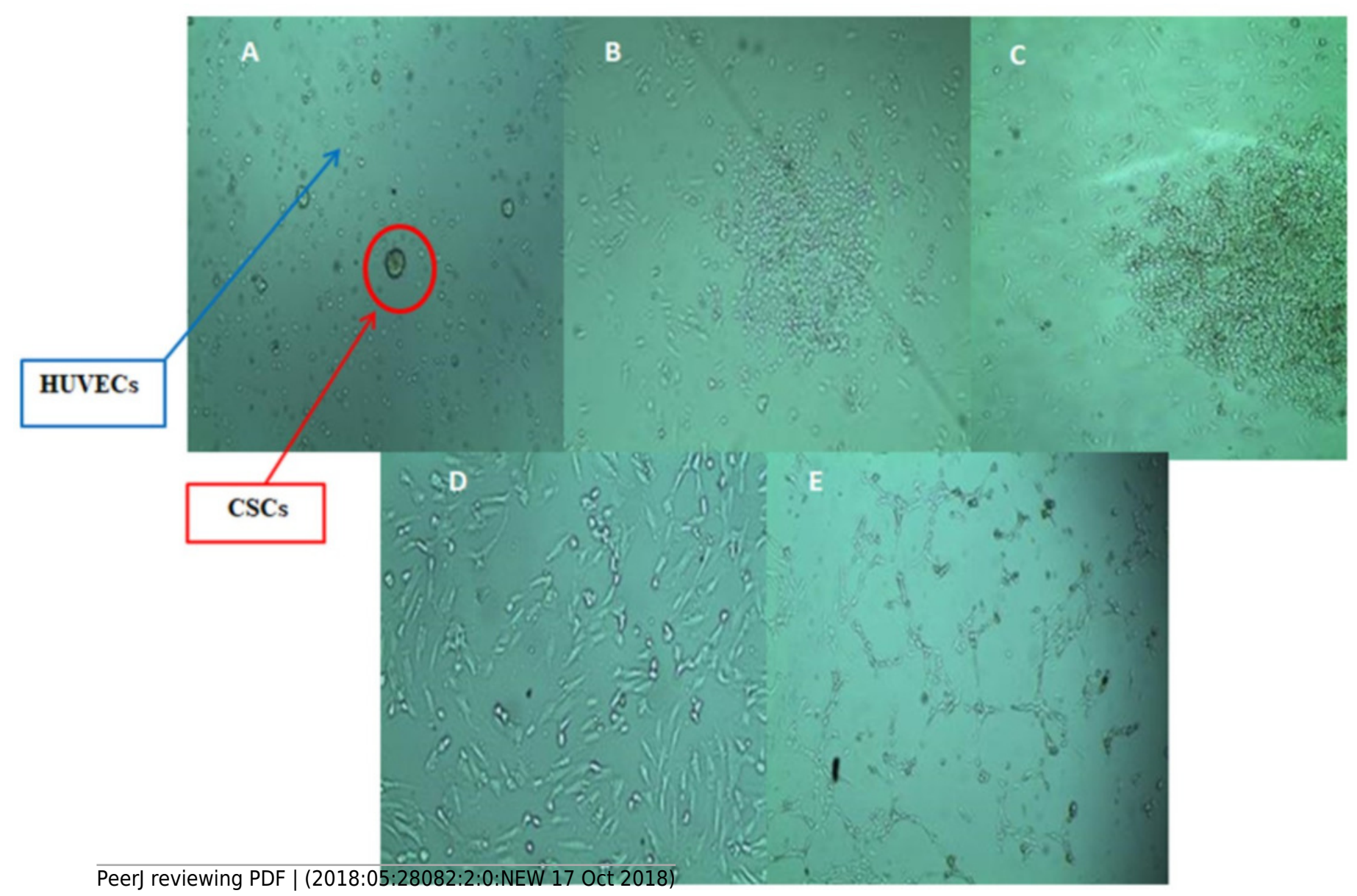




\section{Figure 10}

HUVECs cell migration assessment using wound-healing assay

The upper group of images represents the wound at zero time of treatment with CdM while lower images represent the wound after 48 hours of treatment with CdM. (A) control treated with CdM obtained from CSCs ${ }^{\mathrm{NOR}}$. (B) Treated with CdM from INTR.20 shot. (C) Treated with CdM from CONT.5 shot. Representative images magnification ( $4 x$ objective) Olympus inverted microscope. 

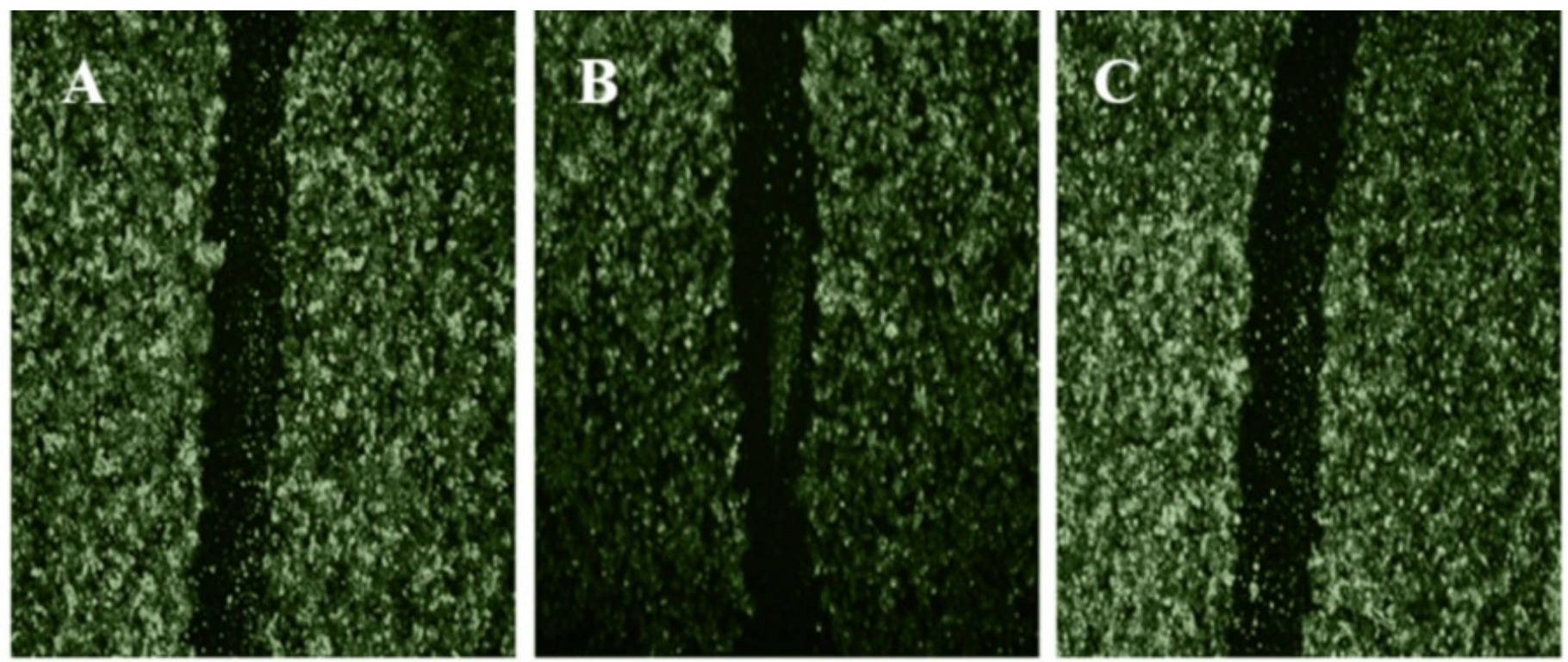

0 Hour
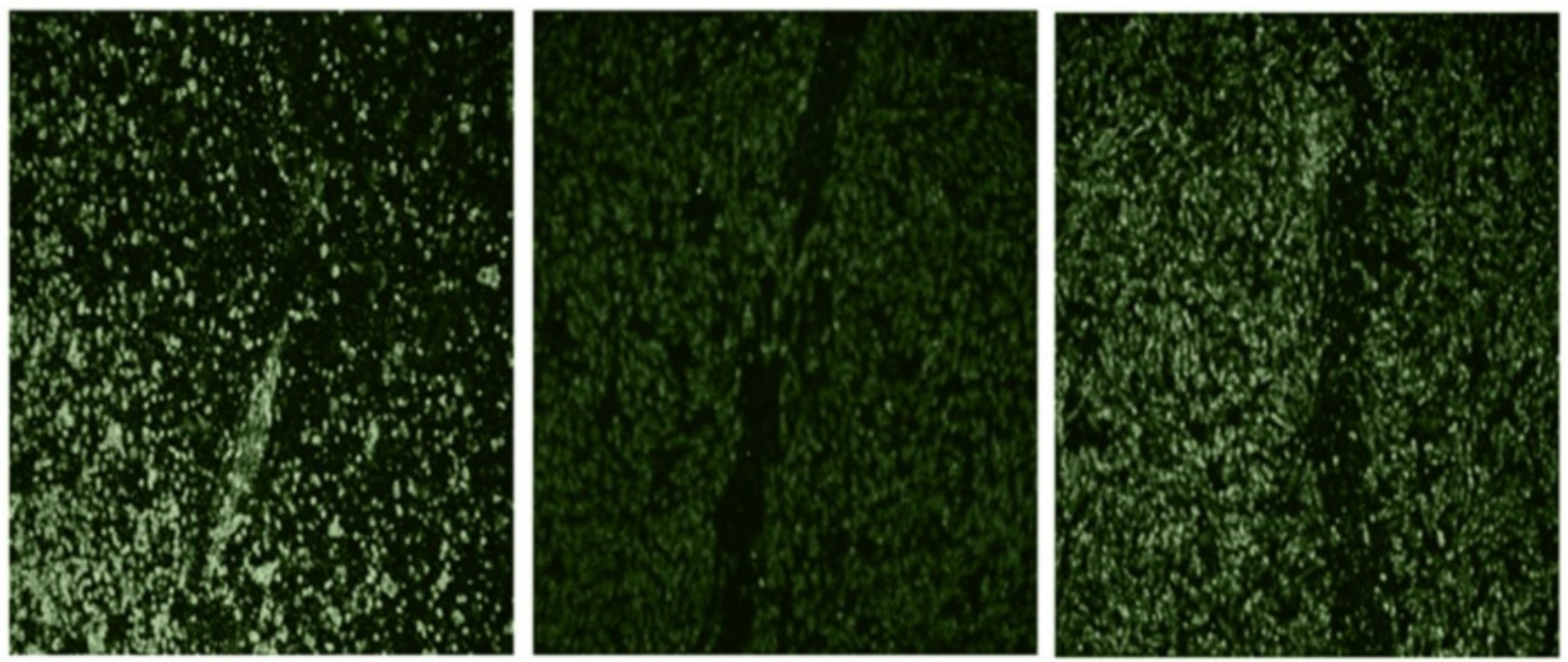

48 Hour

Control

INTR.20

CONT.5 


\section{Figure 11}

Wound healing of CSCS

The upper group of images demonstrate the wound surface area of CSCs monolayer at the time of wound scratching (zero time). The lower group of images are for wound closure after 36 hours from scratching. (A) $\mathrm{CSCS}^{\mathrm{NOR}}$ used as control. (B-H) $\mathrm{CSCS}^{\mathrm{HYP}}$ exposed to various hypoxic treatments. CSCs ${ }^{\text {HYP }}$ that were exposed to INTR.20 shown in (C) and CONT.5 shown in (F) both have had the highest wound closure capacity compared to the control CSCs ${ }^{\mathrm{NOR}}$ in (A).Representative images magnification (4x objective) Olympus inverted microscope. 

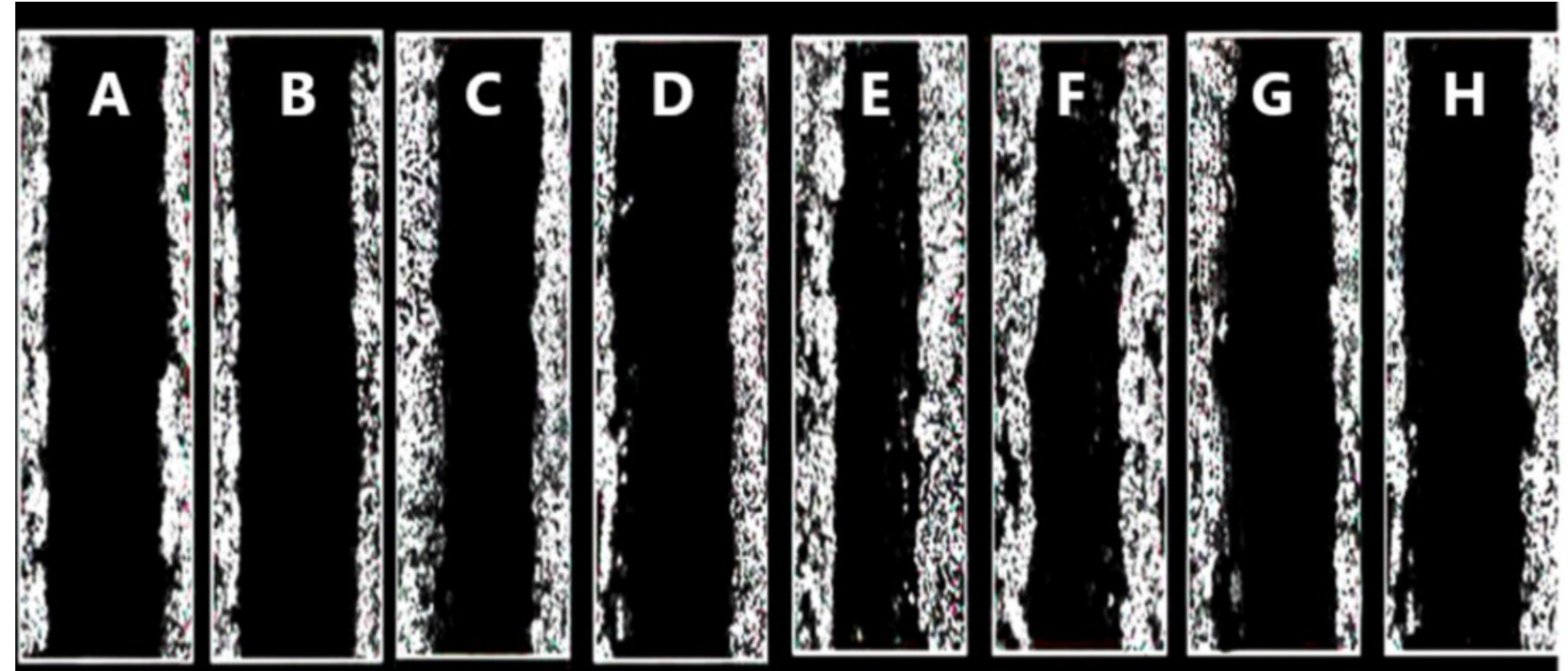

\section{OHour}
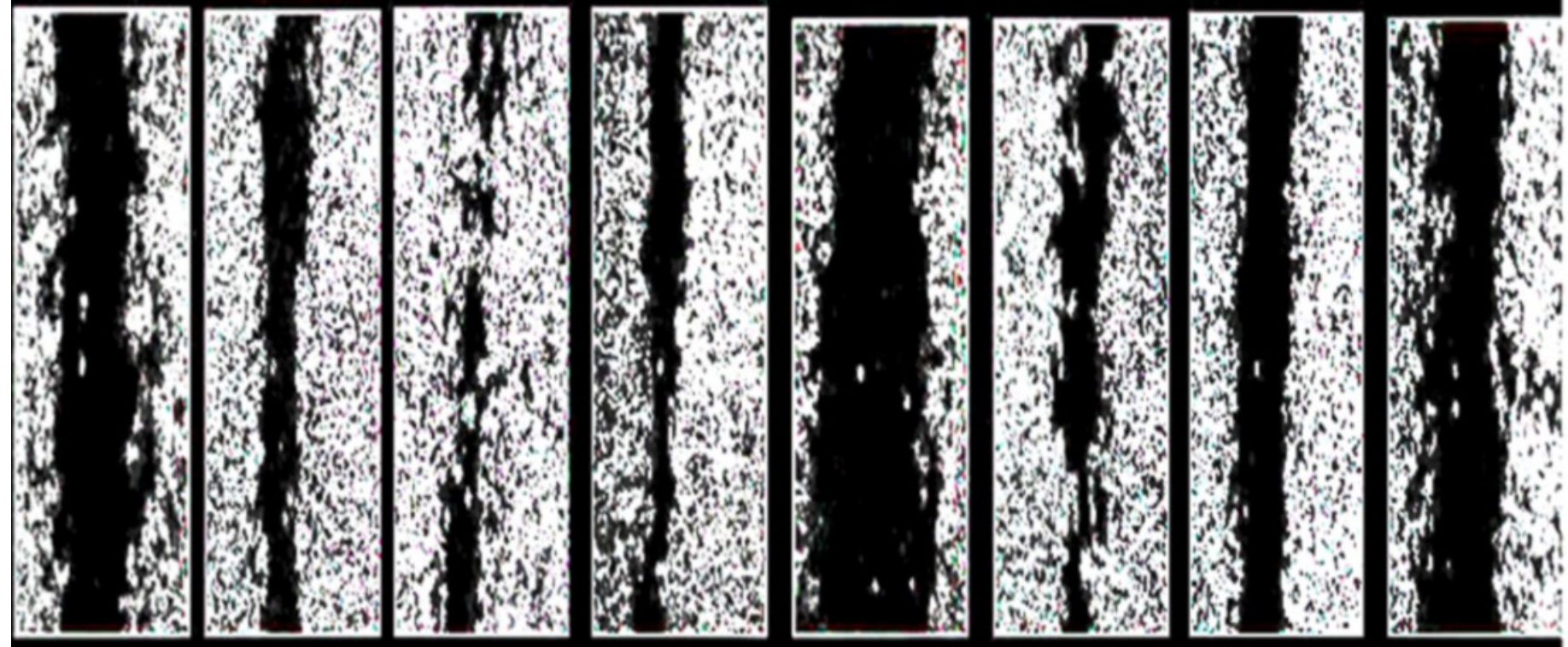

\section{Hour}

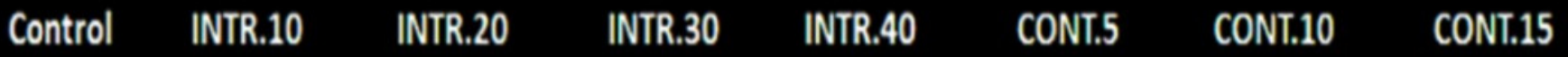




\section{Figure 12}

CSCs wound closure quantification

The quantification of wound closure was measured by ImageJ software and represented in bars expressing the percentage of gap closure. $\mathrm{CSCs}^{\mathrm{HYP}}$ subpopulation that was exposed to INTR.20 or CONT.5 manifested the highest wound closure capacity compared to the control CSCS $^{\text {NOR }}$. After 36 hours, the wound closure was $98 \%$ for INTR.20 and 95\% for CONT.5 whereas $40 \%$ for control. The results represent mean \pm SD of three independent experiments. Statistically significant differences among the treated and control cells are shown by $(*)\left(p^{<}\right.$ $0.001)$ and $(* *)(p<0.0001)$.

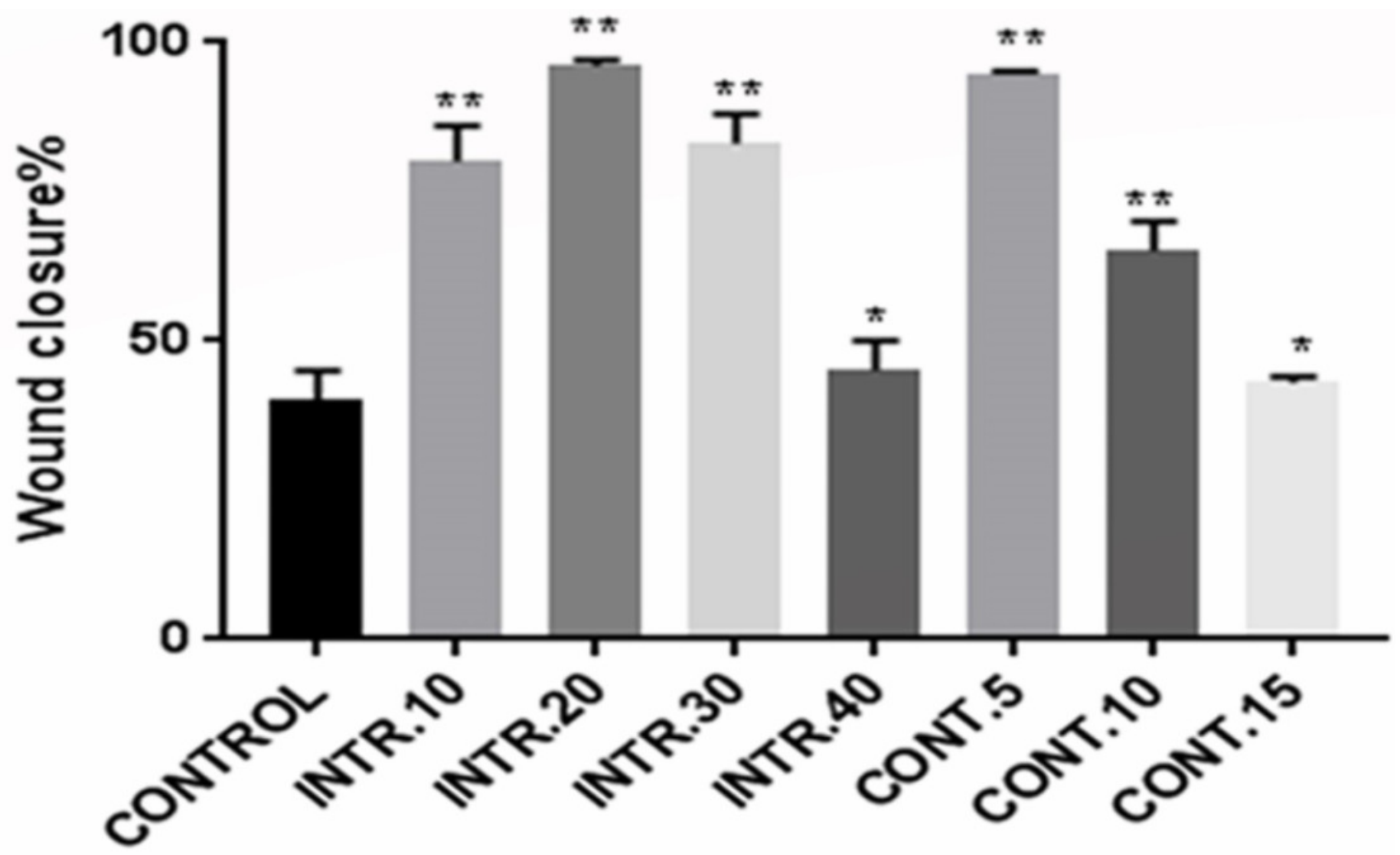




\section{Table $\mathbf{1}$ (on next page)}

The summary of $\mathrm{CD} 44^{+} / \mathrm{CD} 24$ expression obtained by flow cytometry among different MCF-7 sub-population derived cells. 
1

\begin{tabular}{c|c}
\hline \hline Cells Subpopulation & $\begin{array}{c}\text { CD44 }^{+} / \mathbf{C D 2 4}^{-} \\
\text {Expression \% }\end{array}$ \\
\hline \hline Parent MCF-7 Cells & $1.0( \pm 0.1 \%)$ \\
\hline $\begin{array}{c}\text { Unsorted Mammospheres (Day 7 in ultra-low attachment, } \\
\text { serum free media) }\end{array}$ & $33.2( \pm 3.0 \%)$ \\
\hline Sorted CSCs mammospheres (3 days after sorting) & $81.0( \pm 7.5 \%)$ \\
\hline Sorted CSCs mammospheres (After a total of 21 days) & $35.5( \pm 4.5 \%)$ \\
\hline CSCs $^{\mathrm{HYP}}$ exposed to INTR.20 shots & $39.8( \pm 5.2 \%)$ \\
\hline $\mathrm{CSCs}^{\mathrm{HYP}}$ exposed to CONT.5 shots & $51.6( \pm 6.1 \%)$ \\
\hline $\mathrm{CSCs}^{\mathrm{HYP}}$ exposed to INTR.40 shots & $0.3( \pm 0.01 \%)$ \\
\hline $\mathrm{CSCs}^{\mathrm{HYP}}$ exposed to CONT.15 shots & $0.5( \pm 0.05 \%)$ \\
\hline \hline
\end{tabular}




\section{Table 2 (on next page)}

The IC50 values (UM) of doxorubicin against MCF-7 parental, CSCs ${ }^{\text {NOR }}$ mammospheres and $\mathrm{CSCS}^{\mathrm{HYP}}$ of different hypoxic shots after 72 hours treatment. 


\begin{tabular}{|c|c|c|c|}
\hline Cell Subpopulation & $\begin{array}{c}\text { IC50 (uM) } \\
\pm \text { STD }\end{array}$ & $\begin{array}{l}\text { Fold Change Compared } \\
\text { to sorted normoxic } \\
\text { Mammosphere (Control) }\end{array}$ & $\begin{array}{c}\text { Fold Change } \\
\text { Compared to } \\
\text { Parental MCF- } \\
7 \\
\end{array}$ \\
\hline MCF-7 Parental & $0.46( \pm 0.06)$ & $\mathrm{N} / \mathrm{A}^{\dagger}$ & $\mathrm{N} / \mathrm{A}^{\bullet}$ \\
\hline $\mathrm{CSCs}^{\mathrm{NOR}}$ mammospheres (Control) & $1.90( \pm 0.35)$ & $\mathrm{N} / \mathrm{A}^{\vee}$ & $4.08 \uparrow$ \\
\hline $\mathrm{CSCs}^{\mathrm{HYP}}$ exposed to INTR.10 & $4.64( \pm 0.15)$ & $2.45 \uparrow$ & $10.00 \uparrow$ \\
\hline $\mathrm{CSCs}^{\mathrm{HYP}}$ exposed to INTR.20 & $6.2( \pm 0.29)$ & $3.28 \uparrow$ & $13.39 \uparrow$ \\
\hline $\mathrm{CSCs}^{\mathrm{HYP}}$ exposed to INTR.30 & $4.101( \pm 0.22)$ & $2.16 \uparrow$ & $8.84 \uparrow$ \\
\hline $\mathrm{CSCs}^{\mathrm{HYP}}$ exposed to INTR.40 & $0.334( \pm 0.02)$ & $0.18 \downarrow$ & $0.72 \downarrow$ \\
\hline $\mathrm{CSCs}^{\mathrm{HYP}}$ exposed to CONT.5 & $7.124( \pm 0.19)$ & $3.76 \uparrow$ & $15.35 \uparrow$ \\
\hline $\mathrm{CSCs}^{\mathrm{HYP}}$ exposed to CONT.10 & $4.486( \pm 0.11)$ & $2.36 \uparrow$ & $9.67 \uparrow$ \\
\hline $\mathrm{CSCs}^{\mathrm{HYP}}$ exposed to CONT.15 & $0.441( \pm 0.04)$ & $0.23 \downarrow$ & $0.95 \downarrow$ \\
\hline
\end{tabular}

All data are expressed by means $\pm \mathrm{SD}$; $\mathrm{P}<0.0001$ compared to the parental group.

$\checkmark$ N/A: Value not calculated; not applicable to compare the reference to its value

$\dagger \mathrm{N} / \mathrm{A}$ : Value not calculated because we refer and compare the $\mathrm{IC}_{50}$ of sorted $\mathrm{CSCs}^{\mathrm{NOR}}$ mammospheres to MCF-7 Parental cells $\mathrm{IC}_{50}$ not the contrary

(N/A: Not applicable, STD: Standard Deviation, uM: microMolar, $\uparrow:$ Increased, $\downarrow$ : Decrease) 